\title{
Real-time Delay with Network Coding and Feedback
}

\author{
Eleni Drinea \\ Christina Fragouli \\ Lorenzo Keller \\ Swiss Federal Institute of Technology, Lausanne (EPFL) \\ E-mail: \{eleni.drinea, christina.fragouli, lorenzo.keller\}@epfl.ch
}

\begin{abstract}
We consider the problem of minimizing delay when broadcasting over erasure channels with feedback. A sender wishes to communicate the same set of $\mu$ messages to several receivers. The sender can broadcast a single message or a combination (encoding) of messages to all receivers at each timestep, through separate erasure channels. Receivers provide feedback as to whether the transmission was received. If, at some time step, a receiver cannot identify a new message, delay is incurred. Our notion of delay is motivated by real-time applications that request progressively refined input, such as the successive refinement of an image encoded using multiple description coding.

Our setup is novel because it combines coding techniques with feedback information to the end of minimizing delay. Uncoded scheduling or use of erasure correction coding, such as maximum distance separable (MDS) codes, has been well-studied in the literature. We show that our setup allows $\Theta(\mu)$ benefits as compared to both previous approaches for offline algorithms, while feedback allows online algorithms to achieve smaller delay compared to online algorithms without feedback.

Our main complexity results are that the offline minimization problem is $N P$-hard when the sender only schedules single messages and that the general problem remains $N P$-hard even when coding is allowed. However we show that coding does offer complexity gains by exhibiting specific classes of erasure instances that become trivial under coding schemes. We also discuss online heuristics and evaluate their performance through simulations.
\end{abstract}

Keywords: broadcasting, feedback, delay, erasures, linear coding, computational complexity, online scheduling and coding algorithms.

\section{Introduction}

Current and emerging applications, such as satellite imaging, roadside to vehicle communication, internet tv, wireless downlink broadcasting, require content to be downloaded quickly and reliably from a host over possibly unknown channels. In practical networks, transmissions are subject to errors: packets get dropped due to congested links, wireless fading and interference, expired timestamps, etc. Such losses are perceived as packet erasures at higher layers, and are often modeled using independent erasure channels.

To cope with unknown channels, feedback information is often available at the broadcasting source. Thus the source, when deciding what to transmit next, knows which subset of receivers successfully received each of its past transmissions. That is, the source has perfect feedback information. Feedback can be efficiently employed in a wireless environment: the source might acquire such information by taking advantage of the symmetry of wireless links, or by collecting acknowledgment packets explicitly using specifically designed control traffic [10], or implicitly, by overhearing transmissions from the receiver nodes [16]. In satellite transmissions, a satellite might learn when a receiver goes in a deep fade (e.g., enters a tunnel), in which case it loses a sequence of packets. It is also known how to explicitly collect acknowledgments in wired networks, when the source multicasts the same content over a distribution tree in an overlay network [13].

In this paper, we consider the problem of combining coding techniques and feedback information over broadcasting channels to offer reliable content delivery under delay guarantees. Our notion of delay 
is motivated from real-time applications with progressively refined input. Such a paradigm is provided by multiple description coding that we adopt as our illustrating example in the following; however, our notion of delay is relevant to a much more general class of applications.

Multiple description is a well studied data compression technique which allows to provide robustness and graceful recovery in the presence of unknown channel conditions. Although the theoretical problem was already introduced in the 80's (see for example [14]), the research interest in the field was significantly invigorated during the last few years, due to the numerous identified network applications, such as image and video delivery (see for example [25, 9, 3, 26, 27], and [15] for a tutorial paper). The main idea is that we encode our file, for example an image, using a number $\mu$ of equally important descriptions, and each description is send separately to the receiver. Depending on the channel conditions, the receiver may receive a different number of descriptions. These descriptions are constructed to have the following property: if a receiver receives a single description (any one of them), it gets a coarse version of the image that is within some appropriately defined distortion guarantees from the original. If a receiver gets any two descriptions, it can reconstruct the image more accurately. Generally, the larger the number of descriptions, the smaller the distortion distance from the original. Reception of all descriptions results in the most accurate reconstruction. Note that in this construction, it is only the number of different received files that defines the reconstruction accuracy; the ordering at which files are received plays no role.

Consider now an application that requires fast delivery of images over a wireless network, for example from a road-basestation of a transportation network to passing vehicles. Assume that the image is encoded using multiple description, and thus the basestation has $\mu$ blocks to deliver. When communicating towards a single receiver, simple sequential transmission of the blocks suffices: the underlying multiple description coding will determine the image quality experienced by the receiver, as a function of the number different blocks collected. The problem becomes much more challenging when the image needs to be broadcasted to a number of receivers, each of which receives information over its own erasure channel. The sender may use a scheduling algorithm to decide which image block to broadcast next. In this paper, we propose instead to use a coding algorithm, that encode the blocks we need to transmit to the receivers. Both in the case of scheduling or coding, the algorithm may use the feedback information it has collected (i.e., which receivers received the previous transmissions) to decide on the current transmission. Note that our proposed coding is additional to the multiple description data compression: it decides which and how many image blocks it will combine together, and falls in the area of network coding, as its main purpose is to better share the network resources among the contending receivers. Network coding is an emerging area that has attracted a very significant research interest during the last decade, as it promises to revolutionize information flow through networks (see $[1,18]$ and $[12]$ for an introductory tutorial).

Every time receiver $r_{j}$ receives successfully, it wants to learn some missing piece of information, namely any image description it does not know yet. This motivates us to increment the delay $d_{j}$ of $r_{j}$ by one every time $r_{j}$ successfully receives a transmission of the following type: (i) an image description $r_{j}$ already knows, or (ii) an encoding of image descriptions which, when combined with $r_{j}$ 's successful receptions so far, does not allow $r_{j}$ to immediately extract at least one image block $r_{j}$ does not know yet. This definition allows us to disengage delay from the erasure frequency as we only count delay when a transmission is successful. It also allows us to capture two causes of delay: delay due to useless received packets, namely packets that bring duplicate information to their receiver, and delay due to packets that, although useful, do not allow their receiver to immediately decode some unknown message. Finally, our definition of delay is the simplest instantiation possible, as it does not take into account any ordering: we thus hope that a good understanding of this problem can serve as a first step towards more combinatorially demanding delay definitions.

The main questions we consider in this paper are (i) whether coding offers benefits in terms of delay, and (ii) how to design coding schemes that minimize average and maximum delay, and what is the complexity of this task. We focus in the case where all receivers are interested in the same content 
because understanding this simple model offers a first step towards variations, where receivers may demand different subsets of the messages or request the messages in a specific order. It is worth noting that the popular solution of employing rate-less erasure correcting codes at the source such as LT or Raptor codes $[20,28]$ for reliable broadcasting over erasure channels, performs very poorly in terms of delay (see also Subsection 1.1).

Our contributions include the following. Concerning the complexity of the offline problem, we show that minimizing the average and maximum delay when the source uses scheduling is $N P$-hard. We then examine the complexity of the problem when coding is allowed and show that, although specific classes of erasure instances become trivial, the general problem remains $N P$-hard. We examine classes of erasure instances where coding offers significant benefits in terms of delay, and give a simple inapproximability result for maximum offline delay. Finally, we discuss heuristic online algorithms where the erasures of different receivers are independent and i.i.d. distributed. We evaluate the performance of our heuristics through simulations. The latter verify our observation that coding can significantly reduce delay compared to scheduling.

The importance of our work lies perhaps in that, to the best of our knowledge, it is the first to examine the complexity and algorithmic aspects of the joint use of coding and feedback information for delay-optimal content delivery. Erasures are inherent in many realistic networks, and we believe that the trade-off beween rate and delay that arises in our setting is worth exploring further.

The remainder of this paper is organized as follows. Section 2 introduces our model and notation. Section 3 examines the complexity of offline broadcasting with scheduling, while Section 4 examines the complexity when coding at the source is allowed. Section 5 discusses online results. Section 6 concludes.

\section{$1.1 \quad$ Related Work}

A significant body of work has investigated the problem of scheduling user requests over a broadcast medium to maximize the per-user received rate and minimize the response time; see for example $[7,4,8$, 22]. In this setup, users typically arrive at different time instances, and ask for possibly different content. No coding is employed and no errors are assumed. The difficulty of the problem, which was recently shown to be $N P$-hard [7], arises from having to share the common medium over the contenting requests. It is worth mentioning that if receivers were to ask for the same data items (e.g., satellite images) in any order, then even if the requests arrive at different time instances, a periodic (circular) transmission of the data items would suffice.

In uncoded transmissions, maximizing the per user throughput naturally minimizes the delay: the faster information is received, the better. However, in the presence of erasures, uncoded transmission leads to repetitive reception and cannot achieve rates close to the optimal, and thus can also not achieve the optimal delay. On the other hand, when coding is employed, delay and rate may become conflicting requirements. For rate-less codes for example, to operate close to capacity and avoid duplicate receptions, we need to encode at the source across $\mu$ packets, for large values of $\mu[20,28]$. A receiver needs to wait to collect $\Theta(\mu)$ coded packets to be able to decode, which implies a delay of $\Theta(\mu)$. Indeed, in the presence of erasures, satisfying requests even for the same content becomes a challenging problem [11]. In [?, 29] use of MDS codes has also been proposed, but their performance is inferior to Raptor codes both in terms of complexity and adaptability to unknown channel conditions.

Our work can also be viewed as an instantiation of network coding with feedback. Recent work has looked at use of acknowledgments and coding to optimize the achievable rate, under the condition that each received packet is either useless or can be immediately decoded by the destination [16]. Such schemes, although simple to implement, do not offer rate or delay guarantees. Another line of work has looked into use of coding and feedback to minimize the queue size at the sender [30]. This performance metric is quite different from delay. Also, [23] examines use of feedback over broadcast erasure channels to optimize rate and achieve zero probability of error.

Finally, Birk and Kol [6] introduced a related broadcasting scenario, called Index Coding. Index 
Coding was examined in a line of works [5, 19, 2]. A more general setting than Index Coding, recently analyzed by Alon et. al. in [2], is as follows. A source possesses $m$ messages. There are $n$ receivers. Each receiver somehow, e.g., through some initial phase where the source schedules single messages only and every receiver receives these messages through an erasure channel, ends up with a subset of the $m$ messages by some time $t$; this subset is the side information of the receiver. It is then assumed that no receiver experiences any erasures after time $t$, and that each receiver wants exactly one of the source messages it is still missing. The goal is to find the minimum length of the codeword whose transmission will allow all receivers to simultaneously recover the messages they want.

Our problem differs from the formulation above in many ways. First, we do not restrict our model to guarantee that there is some time $t$ after which every receiver will have a perfect communication channel with the source. Second, the source is allowed to transmit linear combinations at any time slot, so even if there was such a time $t$, the side information of a receiver would not just be single messages. More importantly, given that we do not impose any time $t$ after which every receiver successfully receives the source transmissions, it is not difficult to see that applying at every time step an erasure correcting code that allows every successfully receiving receiver to reconstruct one of its missing message does not necessarily minimize delay.

As far as we know, this is the first work that examines jointly optimizing coding and use of feedback information for delay-optimal content delivery. Our paper builds on a preliminary work [17], where we introduced the problem and proposed some online heuristics.

\section{The Model}

Consider a source that wants to convey $\mu$ messages to $\rho$ receivers using broadcast transmissions. Time is slotted, and at the beginning of each time slot $t \geq 1$, the source is allowed to transmit (linear or non-linear) combinations of the messages, which we call packets. We denote the packet transmitted at the beginning of time slot $t \geq 1$ by $p(t)$. We call a broadcasting scheme that schedules single (uncoded) messages at every time step a scheduling scheme. If the scheme is allowed to use coding operations (combinations) on the messages, we call it a coding scheme. Each receiver receives information from the source through an erasure channel, which might range from only deep fadings to i.i.d. erasures and may depend on the erasure channels of other receivers. We denote by $K_{j}^{t} \in\{0,1\}$ the realization of receiver $r_{j}$ 's channel at time $t$ with $K_{j}^{t}=1$ if and only if $r_{j}$ receives $p(t)$. In a worst case model these realizations could have given values, while in a probabilistic setting they would be random variables.

Depending on whether the transmitted packet successfully reached a receiver or not, the receiver sends an ACK or a NACK to the source respectively (we assume that the feedback channels are perfect). We assume that $K_{j}^{t}$ is received by the source at the end of time slot $t$. Therefore the source can use this information for generating the packet $p(t+1)$ transmitted at the next time slot $t+1$. We assume that the duration of the time slot is sufficient for the receivers to receive the packet and decode it (if possible) using the packets they have already received. A receiver who has decoded all $\mu$ messages is no longer interested in the source transmissions. The source transmits a packet during every time slot until all receivers have decoded all messages.

We can think of the $\mu$ source messages as defining a $\mu$-dimensional space over a finite field $\mathbb{F}_{q}$, where each message corresponds to one of the orthonormal basis vectors $\left\{e_{1}, e_{2}, \ldots, e_{\mu}\right\}$. We will denote by $p(t)$ the linear packet ${ }^{1}$ the source transmits at time $t$. Linear packets are of the form $(c, x)$ where $c \in \mathbf{F}_{q}^{\mu}$ and $x=\sum_{j} c_{j} e_{j}$; the choice of the coefficient vector $c$ determines $x$, so we leave $x$ implied in what follows. Operations over a finite field $\mathbf{F}_{q}$ of size say $q=2^{\ell}$ in practice means that we divide the binary packets the source produces into contiguous sets of $\ell$ bits, and treat each such set as a symbol of $\mathbf{F}_{q}$. Linear combining of the packets occurs symbol-wise.

Let $\Pi_{j}^{t}$ be the subspace collected by $r_{j}$ at the end of time slot $t$ and $E_{j}^{t}$ the set of vectors $e_{\ell} \in \Pi_{j}^{t}$.

\footnotetext{
${ }^{1}$ It is not difficult to generalize this discussion to packets that consist of nonlinear combinations of messages.
} 
We say that a received vector (packet) brings novel information to a receiver $r_{j}$ if it increases the dimension of $\Pi_{j}$ by one. A class of schemes that will play an important role henceforth are schemes where every successfully received packet brings innovative information to its receiver. We call these schemes rate-optimal. In a rate-optimal scheme, a receiver $r_{j}$ that has received $\ell$ packets, has collected an $\ell$-dimensional subspace $\Pi_{j}$ of the $\mu$-dimensional space. For $\ell=\mu$, the receiver can successfully decode all source messages. The following properties of rate-optimal schemes are straightforward (also see [17]): 1. A receiver $r_{j}$ can decode the source message $e_{i}$ if and only if $e_{i} \in \Pi_{j}$; and

2. With slight abuse of notation, let $\Pi_{1}$ denote the subspace spanned by the vectors $<p\left(i_{1}\right), \ldots, p\left(i_{t_{1}}\right)>$ receiver $r_{j}$ has collected by time $t_{1}$ (recall that $r_{j}$ may have some erasures during the $t_{1}$ time steps, hence $\left.i_{t_{1}} \leq t_{1}\right)$, and $\Pi_{2}$ the subspace spanned by the vectors $\left\langle p\left(i_{t_{1}}+1\right), \ldots, p\left(i_{t_{2}}\right)>\right.$ the same receiver $r_{j}$ collects between times $t_{1}+1$ and $t_{2}$. If $E_{1}$ is the set of vectors $e_{\ell} \in \Pi_{1}$ and $E_{2}$ the set of vectors $e_{\ell} \in \Pi_{2}$, then $E_{1} \cap E_{2}=\emptyset$, for all $j, t_{1}<\mu$ and $t_{2} \leq \mu$.

Let $\mathbf{1}(\cdot)$ be the indicator function.

Definition 1 The delay $d_{j}^{T}$ that receiver $r_{j}$ experiences when the source uses transmission scheme $T$ is the number of packets that, although successfully received, did not allow $r_{j}$ to immediately decode a new message. ${ }^{2}$ In symbols,

$$
d_{j}^{T} \triangleq 1+\sum_{t:\left|E_{j}^{t}\right|<\mu} \mathbf{1}\left(E_{j}^{t}=E_{j}^{t-1}\right) \cdot K_{j}^{t}
$$

Let $D_{a}^{T}$ and $D_{w}^{T}$ denote the average and worst case delay of transmission scheme $T$ respectively, given by

$$
\mathcal{D}_{a}^{T} \triangleq \frac{1}{\rho} \sum_{1 \leq j \leq \rho} d_{j}^{T}, \quad \text { and } \quad \mathcal{D}_{w}^{T} \triangleq \max _{1 \leq j \leq \rho} d_{j}^{T} .
$$

Different schemes may result in different values for $\mathcal{D}_{a}^{T}$ and $\mathcal{D}_{w}^{T}$. Our goal is to find among all transmission schemes the (possibly different, e.g. , see [17]) transmission schemes under which the average and worstcase delay are minimized.

As an example, given our definition of delay, if a given broadcasting instance allows for the minimum delay of one, the scheme must be rate-optimal or proved rate-optimal because of the realization of the channels. In other words, may have transmitted packets that were not novel for certain receivers during certain time steps but the specific receivers experienced erasures at the specific time steps. On the other hand, if a scheme is not rate optimal, then any successfully received packet that does not bring new information to its receiver, increments the delay of its receiver by one. Hence any scheme that is not rate optimal given the realizations of the channels will have average delay strictly larger than one and maximum delay at least two.

The discussion above implies that further insight into the online problem can be gained by examining its offline version. The offline broadcasting instance has two additional inputs: (a) an integer $\tau$ that stands for the number of source transmissions by which all receivers have received all messages, and (b) a $\tau \times \rho$ symbolic matrix $P$ whose entries take values from $\{\sqrt{ }, \mathrm{x}\} . P$ is defined by the successful receptions and the erasures of the $\rho$ receivers during the $\tau$ time slots, which are known in the offline scenario: entry $P(t, j)=\sqrt{ }$ if and only if receiver $r_{j}$ successfully received the packet $p(t)$ transmitted at time slot $t .{ }^{3}$ We shall henceforth denote an offline broadcasting instance by the quadruple $(\mu, \rho, \tau, P)$, and refer to $P$ as the erasure matrix of the instance.

We say that a broadcasting scheme for the source completes the offline instance $(\mu, \rho, \tau, P)$ if by time $\tau$, all receivers have decoded all messages. Observe that a necessary and sufficient condition for a scheme to complete the offline instance is that every receiver has at least $\mu$ successful receptions by time $\tau$. For

\footnotetext{
${ }^{2}$ We introduce the +1 in the delay for technical reasons -we can interpret this as setup time: e.g., the time slot $t=0$ is used by the source to identify the number of receivers in the system.

${ }^{3}$ We introduce $P(t, j)$ for the offline scenario while we use $K_{j}^{t}$ in the online scenario.
} 
example, any rate-optimal scheme requires exactly $\mu$ successful receptions per receiver to guarantee that all receivers can decode all messages, regardless of the delay.

Offline analysis is useful because it can be used as a benchmark for the online algorithms: the performance of the optimal offline algorithm lower bounds the performance of any online algorithm. Moreover, offline problems can be particularly interesting and challenging on their own, as the works on Index Coding $[6,5,19,2]$ show.

\section{Minimizing Delay with Scheduling Schemes is NP-hard}

Given an offline broadcasting instance $(\mu, \rho, \tau, P)$, the scheduling problem we are considering is to minimize the average (maximum) delay under any scheduling scheme that completes the instance. Observe that a priori this appears to be an easier problem than the one studied in [7] since our notion of delay is relaxed as all receivers need all messages and not just specific subsets of messages, and further, the order in which the messages are received does not matter.

The decision version of the optimization problem above has as an extra input an integer $d \geq 1$, and answers "yes" if and only if there is a scheduling scheme that completes $(\mu, \rho, \tau, P)$ with total (maximum) delay at most $d^{4}$. An algorithm that solves the minimization problem for total (maximum) delay should be able to answer the decision problem for every value of $d$. Since the minimum possible value for both average and maximum delay is one (the delay corresponding to the initialization phase), and since average delay of one implies maximum delay of one, it suffices to prove that it is hard to decide if the average delay is one, in order to prove that both minimization problems are NP-hard. This is the main result of this section and it is summarized in the following theorem.

Theorem 1 Minimizing average and maximum delay in offline broadcasting in the presence of erasures and when the source uses scheduling schemes is NP-hard.

In the rest we will prove Theorem 1 by reducing 3SAT to average delay of one in offline broadcasting. We shall henceforth refer to average delay simply as delay.

Given a formula $\phi$ in CNF on $n$ variables $x_{1}, \ldots, x_{n}$, and $m$ clauses $c_{1}, \ldots, c_{m}$, where each clause consists of disjunctions of exactly 3 literals, we want to decide if there exists an assignment of truth values to the variables such that all clauses are satisfied.

We will construct an offline broadcasting instance $B(\phi)=(\mu, \rho, \tau, P(\phi))$ such that $\phi$ is satisfiable if and only if there is a scheduling scheme that completes $B(\phi)$ with delay one. In our instance, the source has $\mu=2 n$ messages, there are $\rho=n+2 m$ receivers, and $\tau=4 n+5 m$ time slots. Our construction guarantees that each receiver has exactly $\mu=2 n$ successful receptions by time $\tau$. Notice that this choice of $\tau$ suffices to decide if there is a delay-one scheme for our instance: any such scheme has to be rate-optimal and therefore must deliver all $\mu$ messages to every receiver during the first $\mu$ successful receptions of the receiver starting at $t=1$.

In more detail, our construction works as follows. For every variable $x_{i}, 1 \leq i \leq n$ we introduce 2 messages, $e_{i}$ and $\bar{e}_{i}$. One receiver $D^{i}$ is introduced for every variable $x_{i}$ (their role will be discussed after the construction of $P(\phi)$ is complete). Also, two receivers, $C_{1}^{j}$ and $C_{2}^{j}$ are introduced for every clause $c_{j}$, $1 \leq j \leq m$. This results in the erasure matrix $P(\phi)$ having $\rho=n+2 m$ columns.

We now move on to discussing the number of rows in $P(\phi)$. For every variable $x_{i}$, we introduce 4 consecutive time slots, which we call the variable period $\beta_{i} ; \beta_{i}$ starts at time slot $4(i-1)+1$, and ends at time slot $4 i$. Following the $n$-th variable period, we introduce $m$ consecutive clause periods: the $j$-th clause period, denoted by $\gamma_{j}$, consists of 5 time slots, starts at time slot $4 n+5(j-1)+1$, and ends at time slot $4 n+5 j$. Hence $P(\phi)$ has $\tau=4 n+5 m$ rows.

To complete our construction, we need to assign values to the $\tau \cdot \rho$ entries of $P(\phi)$. We will do this sequentially in time, i.e., by first considering the variable periods and then the clause periods.

\footnotetext{
${ }^{4}$ Minimizing total delay is equivalent to minimizing average delay when $m$ is independent of $n$.
} 


\begin{tabular}{|c|c|c|}
\hline Time slot & $C_{1}^{j}$ & $C_{2}^{j}$ \\
\hline $4(i-1)+1$ & $\sqrt{ }$ & $\mathrm{x}$ \\
$4(i-1)+2$ & $\mathrm{x}$ & $\mathrm{x}$ \\
$4(i-1)+3$ & $\mathrm{x}$ & $\sqrt{ }$ \\
$4 i$ & $\mathrm{x}$ & $\mathrm{x}$ \\
\hline
\end{tabular}

\begin{tabular}{|c|c|c|}
\hline Time slot & $C_{1}^{j}$ & $C_{2}^{j}$ \\
\hline $4(i-1)+1$ & $\sqrt{ }$ & $\mathrm{x}$ \\
$2(i-1)+2$ & $\mathrm{x}$ & $\mathrm{x}$ \\
$4(i-1)+3$ & $\mathrm{x}$ & $\mathrm{x}$ \\
$4 i$ & $\mathrm{x}$ & $\sqrt{ }$ \\
\hline
\end{tabular}

\begin{tabular}{|c|c|c|}
\hline Time slot & $C_{1}^{j}$ & $C_{2}^{j}$ \\
\hline $4(i-1)+1$ & $\sqrt{ }$ & $\sqrt{ }$ \\
$4(i-1)+2$ & $\sqrt{ }$ & $\sqrt{ }$ \\
$4(i-1)+3$ & $\mathrm{x}$ & $\mathrm{x}$ \\
$4 i$ & $\mathrm{x}$ & $\mathrm{x}$ \\
\hline
\end{tabular}

Table 1: Erasure patterns for receivers $C_{1}^{j}, C_{2}^{j}$ during $\beta_{i}$. If clause $c_{j}$ contains $x_{i}$, they receive as in the left table; if $c_{j}$ contains $\bar{x}_{i}$, they receive as in the middle table; else $\left(c_{j}\right.$ does not contain $x_{i}$ or $\left.\bar{x}_{i}\right)$, they receive as in the right table.

\begin{tabular}{|c|c|c|c|c|c|c|c|}
\hline Time slot & $D^{1}$ & $\ldots$ & $D^{i-1}$ & $D^{i}$ & $D^{i+1}$ & $\ldots$ & $D^{n}$ \\
\hline $4(i-1)+1$ & $\sqrt{ }$ & $\ldots$ & $\sqrt{ }$ & $\mathrm{x}$ & $\sqrt{ }$ & $\ldots$ & $\sqrt{ }$ \\
$4(i-1)+2$ & $\sqrt{ }$ & $\ldots$ & $\sqrt{ }$ & $\mathrm{x}$ & $\sqrt{ }$ & $\ldots$ & $\sqrt{ }$ \\
$4(i-1)+3$ & $\mathrm{x}$ & $\ldots$ & $\mathrm{x}$ & $\sqrt{ }$ & $\mathrm{x}$ & $\ldots$ & $\mathrm{x}$ \\
$4 i$ & $\mathrm{x}$ & $\ldots$ & $\mathrm{x}$ & $\sqrt{ }$ & $\mathrm{x}$ & $\ldots$ & $\mathrm{x}$ \\
\hline
\end{tabular}

\begin{tabular}{|c|c|c|}
\hline Time slot & $C_{1}^{j}$ & $C_{2}^{j}$ \\
\hline $4 n+5(j-1)+1$ & $\sqrt{ }$ & $\sqrt{ }$ \\
$4 n+5(j-1)+2$ & $\mathrm{x}$ & $\sqrt{ }$ \\
$4 n+5(j-1)+3$ & $\mathrm{x}$ & $\sqrt{ }$ \\
$4 n+5(j-1)+4$ & $\sqrt{ }$ & $\mathrm{x}$ \\
$4 n+5 j$ & $\sqrt{ }$ & $\mathrm{x}$ \\
\hline
\end{tabular}

Table 2: The left table shows receptions of $D^{1}, \ldots, D^{n}$ during $\beta_{i}$. The right table shows receptions of $C_{1}^{j}, C_{2}^{j}$ during clause period $\gamma_{j}$ (all other receivers experience erasures during $\gamma_{j}$ ).

During variable period $\beta_{i}$, for all $1 \leq j \leq m$, receivers $C_{1}^{j}, C_{2}^{j}$ corresponding to clause $c_{j}$ receive as shown in Table 1 depending on whether $x_{i}, \bar{x}_{i}$ or none of them appears in $c_{j}$. Also, during $\beta_{i}$, receivers $D^{\ell}$ for $1 \leq \ell \leq n$, receive as shown in Table 2: for $\ell \neq i, D^{\ell}$ receives during the first two time slots of $\beta_{i}$, while $D^{i}$ receive during the last two time slots.

During clause period $\gamma_{j}$, receivers $C_{1}^{j}, C_{2}^{j}$ corresponding to clause $c_{j}$ receive as shown in the right table of Table 2. All other receivers experience erasures during $\gamma_{j}$.

The above completes our construction. Table 4 in the Appendix shows $P(\phi)$ for the example formula $\phi=\left(x_{1} \vee x_{2} \vee x_{3}\right) \wedge\left(\bar{x}_{1} \vee \bar{x}_{2} \vee x_{3}\right) \wedge\left(\bar{x}_{2} \vee \bar{x}_{3} \vee x_{4}\right)$ for which $B(\phi)=(8,10,31, P(\phi))$.

Some remarks are appropriate at this point. First it is trivial to check that the reduction can be carried out by a deterministic Turing machine in logarithmic space, and that every receiver has exactly $\mu$ successful receptions. So a priori there could be a scheduling scheme completing $B(\phi)$ with delay one. The role of the receivers $D^{i}$ is to guarantee that exactly 2 messages are sent during each $\beta_{i}$, with the two messages sent during the first two time slots being rescheduled during the last two time slots, in any order (see Proposition 2 for a proof). In effect, this flexibility in the scheduling of the messages during the last two time slots of each $\beta_{i}$ is our choice gadget. Our consistency gadget is that during $\beta_{i}$, $C_{2}^{j}$ receives a different message from $C_{2}^{\ell}$ when $x_{i}$ appears in clause $c_{j}$ and $\bar{x}_{i}$ in $c_{\ell}$. Finally our clause constraint gadget is the simultaneous reception of the two receivers corresponding to clause $c_{j}$ during the first time slot of $\gamma_{j}$.

We now move to showing that $\phi$ is satisfiable if and only if $B(\phi)$ admits delay one. Before, we introduce the following two schedulings which will prove useful for our arguments.

Scheduling 1 for variable period $\beta_{i}$ : the ordered sequence of messages $e_{i}, \bar{e}_{i}, e_{i}, \bar{e}_{i}$.

Scheduling 2 for variable period $\beta_{i}$ : the ordered sequence of messages $e_{i}, \bar{e}_{i}, \bar{e}_{i}, e_{i}$.

Proposition 1 If $\phi$ is satisfiable, then there is a scheduling scheme $T_{S}$ that satisfies the offline broadcasting instance $B(\phi)=(2 n, 2 m+n, 4 n+5 m, P(\phi))$ with delay one.

Proof. Consider a satisfying truth assignment for $\phi$. For $1 \leq i \leq n$, if $x_{i}$ is true, $T_{S}$ applies Scheduling 1 for $\beta_{i}$ during $\beta_{i}$, else if $x_{i}$ is false, $T_{S}$ applies Scheduling 2 for $\beta_{i}$ during $\beta_{i}$. Then the first $4 n$ transmissions of $T_{S}$ incur delay one, and $D^{1}, \ldots, D^{n}$ obtain all messages. 
Since $\phi$ is satisfiable, every clause has at least one literal that is true. W.l.o.g., let $c_{j}=\left(\ell_{i} \vee \ell_{a} \vee \ell_{b}\right)$ be any clause, where $\ell_{y}$ is either $x_{y}$ or $\bar{x}_{y}$, and suppose that $\ell_{i}$ is (one of) the satisfying literal(s) for this clause, i.e., $x_{i}$ is set to true if and only $\ell_{i}=x_{i}$. We now show how $T_{S}$ completes the clause periods so that the clause receivers obtain all messages without delay.

By time $4 n$, receivers $C_{1}^{j}, C_{2}^{j}$ know $2 n-6$ messages, i.e., all messages corresponding to the variables that do not appear in clause $c_{j}$. Further (see Table 1 ), $C_{1}^{j}$ knows $\left\{e_{i}, e_{a}, e_{b}\right\}$, and $C_{2}^{j}$ knows exactly one from $\left\{e_{a}, \bar{e}_{a}\right\}$ and one from $\left\{e_{b}, \bar{e}_{b}\right\} . C_{2}^{j}$ also knows $e_{i}$ which he received at the third time slot of $\beta_{i}$ if $x_{i}$ appears in $c_{j}$ (in which case, since $\ell_{i}$ is the satisfying literal of $c_{j}, x_{i}$ was set to true, and $T_{S}$ applied Scheduling 1 for $\beta_{i}$ during $\beta_{i}$ ), or at the fourth time slot of $\beta_{i}$ if $\bar{x}_{i}$ appears in $c_{j}$ (in which case, $x_{i}$ was set to false and $T_{S}$ applied Scheduling 2). Then during the first time slot of $\gamma_{j}$ (see Table 2), $T_{S}$ sends $\bar{e}_{i}$. Next, $T_{S}$ sends $e_{a}$ or $\bar{e}_{a}$, depending on which one $C_{2}^{j}$ missed during $\beta_{a}$; similarly for $e_{b}$ during the third time slot. Finally $T_{s}$ schedules $\bar{e}_{a}$ and $\bar{e}_{b}$ during the last two slots of $\gamma_{j}$. Since these transmissions result in $C_{1}^{j}, C_{2}^{j}$ obtaining all messages without delay, $T_{S}$ satisfies $B(\phi)$ with delay one.

Conversely, let $T_{S}^{\prime}$ be any scheduling scheme that satisfies $B(\phi)$ with delay one. We will exhibit a satisfying truth assignment for $\phi$. We first introduce some notation: for $1 \leq t_{1} \leq t_{T} \leq 4$, we define $E_{i}^{t_{1} \ldots t_{T}}$ to be the (simple) set of the messages scheduled at the discrete time steps $t_{1}, \ldots, t_{T}$ of $\beta_{i}$. For example, $E_{i}^{123}$ is the simple set of messages sent during the first 3 slots of $\beta_{i}$. We can now show a technical but useful lemma concerning properties of $T_{S}^{\prime}$ during the variable periods.

Proposition 2 Consider any scheduling scheme $T_{S}^{\prime}$ that satisfies $B(\phi)$ with delay one. For $1 \leq i \leq n$, $T_{S}^{\prime}$ schedules exactly two new messages during $\beta_{i}$, with the messages sent during the first two time slots of $\beta_{i}$ being resent (in some order) during the last two time slots. In symbols, for all $1 \leq i \neq j \leq n$, $E_{i}^{12}=E_{i}^{34}$ and $E_{i}^{12} \cap E_{j}^{12}=\emptyset$.

Proof. Trivially, $T_{S}^{\prime}$ is rate-optimal for every $D^{i}$ since it satisfies $B(\phi)$ with delay one. Thus $\left|E_{i}^{12} \cup E_{i}^{34}\right| \geq$ 2 for all $i$. Now suppose that there is a $k$ such that $E_{k}^{12} \neq E_{k}^{34}$. It follows that $\left|E_{k}^{12} \cup E_{k}^{34}\right| \geq 3$, so by the pigeonhole principle, there is a message $m$ that was scheduled during both $\beta_{k}$ and some $\beta_{j}$ with $j \neq k$. During $\beta_{k}, m$ was either received by all $D^{\ell}$ with $\ell \neq k$ or by $D^{k}$ or both. During $\beta_{j}, m$ was either received by all $D^{i}$ with $i \neq j$ or by $D^{j}$ or both. If $m$ was received by all $D^{\ell}$ and all $D^{i}$, all $D^{y}$ with $y \neq k, j$ receive $m$ twice (in $\beta_{k}$ and in $\beta_{j}$ ). If $m$ was received by $D^{k}$ and all $D^{i}$, then $D^{k}$ received $m$ twice. If $m$ was received by all $D^{\ell}$ and $D^{j}$, then $D^{j}$ received $m$ twice. Since $T_{S}^{\prime}$ does not introduce delay, we conclude that none of these scenarios may have happened and it must be that $m$ was received by $D^{k}$ in $\beta_{k}$ and by $D^{j}$ in $\beta_{j}$. Then $m \in E_{k}^{34} \cap E_{j}^{34}$. Since $T_{S}^{\prime}$ is rate-optimal for $D^{k}$ and $D^{j}$, it follows that $\left|E_{1}^{12} \cup \ldots \cup E_{k-1}^{12} \cup E_{k}^{34} \cup E_{k+1}^{12} \cup \ldots \cup E_{n}^{12}\right|=2 n$, and $\left|E_{1}^{12} \cup \ldots \cup E_{j-1}^{12} \cup E_{j}^{34} \cup E_{j+1}^{12} \cup \ldots \cup E_{n}^{12}\right|=2 n$, hence $E_{j}^{12} \cup E_{k}^{34}=E_{j}^{34} \cup E_{k}^{12}$. Since $T_{S}^{\prime}$ is also rate-optimal for every $D^{i}$ with $i \neq j, k$, we have that $E_{j}^{12} \cap E_{k}^{12}=\emptyset\left(D^{i}\right.$ receives $E_{j}^{12}$ in $\beta_{j}$ and $E_{k}^{12}$ in $\left.\beta_{k}\right)$. We arrive at a contradiction: if $m \in E_{k}^{34} \cap E_{j}^{34}$, it is impossible that $E_{j}^{12} \cup E_{k}^{34}=E_{j}^{34} \cup E_{k}^{12}$ when $E_{j}^{12} \cap E_{k}^{12}=\emptyset$. Hence $m$ cannot be in $E_{k}^{34} \cap E_{j}^{34}$, implying that for all $k, E_{k}^{12}=E_{k}^{34}$.

W.l.o.g., assume that $T_{S}^{\prime}$ schedules the two messages $e_{x_{i}}, e_{y_{i}}$ during the first two time slots of $\beta_{i}$, in this order. By Proposition 2, these messages will not be rescheduled before time $4 n$, so for the sake of clarity, we may relabel them as $e_{i}, \bar{e}_{i}$ respectively. We define the following truth assignment. For $1 \leq i \leq n$, if $T_{S}^{\prime}$ applied Scheduling 1 for $\beta_{i}$ during $\beta_{i}, x_{i}$ is set to true, else if $T_{S}^{\prime}$ used Scheduling 2 for $\beta_{i}$ during $\beta_{i}, x_{i}$ is set to false. Notice that Proposition 2 guarantees that any $T_{S}^{\prime}$ indeed applied one of these two schedulings during $\beta_{i}$.

We are now ready to conclude the converse direction of our reduction after stating one more proposition whose proof will appear shortly.

Proposition 3 Let $c_{j}=\left(\ell_{i} \vee \ell_{a} \vee \ell_{b}\right)$ be any clause. Any $T_{S}^{\prime}$ that satisfies $B(\phi)$ with delay one is such that $C_{2}^{j}$ has received at least one of $e_{i}, e_{a}, e_{b}$ by time $4 n$. 
Corollary 1 If $T_{S}^{\prime}$ is a scheduling scheme that satisfies $B(\phi)$ with delay one then $\phi$ is satisfiable.

Proof. Consider any clause $c_{j}=\left(\ell_{i} \vee \ell_{a} \vee \ell_{b}\right)$. By Proposition 3, any $T_{S}^{\prime}$ is such that $C_{2}^{j}$ has received at least one of $e_{i}, e_{a}, e_{b}$ by time $4 n$. W.l.o.g., assume $C_{2}^{j}$ received $e_{i}$. If $C_{2}^{j}$ received this message at time $4(i-1)+3$, then $x_{i}$ appears in $c_{j}$ and $T_{S}^{\prime}$ used Scheduling 1 for $\beta_{i}$. Hence our truth assignement set $x_{i}$ to true. Otherwise, if $C_{2}^{j}$ received $e_{i}$ at time $4 i$, then $\bar{x}_{i}$ appears in $c_{j}$ and $T_{S}^{\prime}$ used Scheduling 2 for $\beta_{i}$. Hence our truth assignement set $x_{i}$ to false. In either case, our truth assignment for $x_{i}$ satisfies $c_{j}$. Since Proposition 3 applies to all $C_{2}^{j}$ for $1 \leq j \leq m$, there is (at least) one literal in every clause that is set to true by our truth assignment. Hence $\phi$ is satisfiable.

We now give the proof of Proposition 3.

Proof. Consider the 2 receivers $C_{1}^{j}, C_{2}^{j}$ corresponding to $c_{j}$. By Proposition 2 , under any $T_{S}^{\prime}$, at the beginning of $\gamma_{j}$, each of $C_{1}^{j}, C_{2}^{j}$ knows the $2 n-6$ messages that correspond to the $n-3$ variables that do not appear in $c_{j}$. Further, $C_{1}^{j}$ knows $\left\{e_{i}, e_{a}, e_{b}\right\}$, and $C_{2}^{j}$ knows exactly one of $\left\{e_{i}, \bar{e}_{i}\right\}$, one of $\left\{e_{a}, \bar{e}_{a}\right\}$, and one of $\left\{e_{b}, \bar{e}_{b}\right\}$.

Suppose that $C_{2}^{j}$ received $\left\{\bar{e}_{i}, \bar{e}_{a}, \bar{e}_{b}\right\}$. Then at the first slot of $\gamma_{j}$ where he receives simultaneously with $C_{1}^{j}$, there is no way to avoid delay since every message needed by one receiver incurs delay to the other. However if $C_{2}^{j}$ had received at least one of $e_{i}, e_{a}, e_{b}$, say $e_{i}$, then at the first time slot of $\gamma_{j}, T_{S}^{\prime}$ could schedule $\bar{e}_{i}$ which does not delay any receiver.

\section{Benefits and Limits of Coding in Reducing Complexity}

We here start by attempting to understand what are structural properties of instances where use of offline scheduling results in delay greater than one. We will then show that use of coding across messages can offer two benefits: (i) Reduce the delay. For example, we will see that there are instances where with coding we can have delay one, while with scheduling we cannot. (ii) Reduces the complexity of solving the problem for several cases. For example, for the erasure pattern in Section 3, we can trivially achieve average and maximum delay one: during $\beta_{i}$, with $1 \leq i \leq n$, send $e_{i}, \bar{e}_{i}, e_{i}, \bar{e}_{i}$, while during $\beta_{j}$ for clause $c_{j}$, with $1 \leq j \leq m$, send $e_{i}+\bar{e}_{i}$, then whatever is missing from $C_{2}^{j}$, and finally $\bar{m}_{a}, \bar{m}_{b}$. The main prupose of this section is to examine whether and how much use of coding can help.

We will use the following notation. Let $B_{t}$ denote the set of messages the source has transmitted up to time $t$ and $\bar{B}_{t}$ the set of remaining messages. For receiver $r_{j}$, let $E_{j}^{t}$ denote the set of messages from $B_{t}$ that $r_{j}$ has received, and $\bar{E}_{j}^{t}$ the messages from $B_{t}$ it has not. That is, $B_{t}=E_{j}^{t} \cup \bar{E}_{j}^{t}$ for all $r_{j}$.

For the case of one receiver, trivially, scheduling achieves delay one. For the case of two receivers, we can use the following simple algorithm to ensure delay one: if at time time $t$ (i) both $r_{1}$ and $r_{2}$ receive, transmit a message from $\bar{B}_{t}$ (ii) only $r_{j}$ receives, if $\bar{E}_{j}^{t} \neq \emptyset$ transmit a message from $\bar{E}_{j}^{t}$, otherwise a message from $\bar{B}_{t}$. This scheme ensures that at each time $t$ either $\bar{E}_{1}^{t}=\emptyset$ or $\bar{E}_{2}^{t}=\emptyset$; moreover, $\bar{B}_{t}=\emptyset$ only when at least one of the two receivers has received all messages.

For the case of three receivers, offline scheduling can result in worst case delay of $\mathcal{O}(\mu)$. Indeed, note that for scheduling, delay is introduced only when the transmission scheme cannot be rate optimal. For the erasure pattern in Table 3, assume that each line is repeated for $\mu / 2$ time slots. Rate optimality for $r_{3}$ implies that at $t=\mu+1, \bar{E}_{1}^{t} \cap \bar{E}_{2}^{t}=\emptyset$ and thus, the transmissions at time-slots $t=\mu+1, \ldots, 3 \mu / 2$ will incur sum delay $\mu / 2$ for $r_{1}$ and $r_{2}$. The existence of receiver $r_{3}$ is necessary to ensure that $\bar{E}_{1}^{t} \cap \bar{E}_{2}^{t}=\emptyset$ occurs in offline.

The following straightforward proposition formalizes this observation.

Proposition 4 If at time $t$ there exist receivers $r_{i}$ and $r_{j}$ such that $\bar{E}_{i}^{t} \cap \bar{E}_{j}^{t}=\emptyset$, and following time $t$, for the next $D$ timeslots that $r_{i}$ succesfully receives so does $r_{j}$, with $D \triangleq \min \left\{\left|\bar{E}_{i}^{t}\right|, \quad\left|\bar{E}_{j}^{t}\right|\right\}$, then offline scheduling results in delay $\mathcal{O}(D)$. 


\begin{tabular}{|c|c|c|c|}
\hline time-slots & $r_{1}$ & $r_{2}$ & $r_{3}$ \\
\hline$t=1, \ldots, \mu / 2$ & $\sqrt{ }$ & $\mathrm{x}$ & $\sqrt{ }$ \\
$t=\mu / 2+1, \ldots, \mu$ & $\mathrm{x}$ & $\sqrt{ }$ & $\sqrt{ }$ \\
$t=\mu+1, \ldots, 3 \mu / 2$ & $\sqrt{ }$ & $\sqrt{ }$ & $\mathrm{x}$ \\
\hline
\end{tabular}

\begin{tabular}{|c|c|c|c|c|}
\hline time-slots & $r_{1}$ & $r_{2}$ & $r_{3}$ & $r_{4}$ \\
\hline$t=1, \ldots, \mu / 2$ & $\sqrt{ }$ & $\mathrm{x}$ & $\sqrt{ }$ & $\mathrm{x}$ \\
$t=\mu / 2+1, \ldots, \mu$ & $\mathrm{x}$ & $\sqrt{ }$ & $\sqrt{ }$ & $\mathrm{x}$ \\
$t=\mu+1, \ldots, 3 \mu / 2$ & $\sqrt{ }$ & $\sqrt{ }$ & $\mathrm{x}$ & $\sqrt{ }$ \\
\hline
\end{tabular}

Table 3: The left table gives an erasure pattern where scheduling incurs delay $\mathcal{O}(\mu)$ but coding achieves delay one; the right table gives an erasure pattern where coding as well incurs delay $\mathcal{O}(\mu)$.

Use of coding allows to make the source transmissions rate optimal, for all possible erasure patterns. For example, for the pattern in the left Table 3, it is sufficient at time-slots $t=\mu+1, \ldots, 3 \mu / 2$ to transmit $\mu / 2$ messages from $\bar{E}_{1}^{t}+\bar{E}_{2}^{t}$. However, in this case delay is introduced, if a receiver cannot decode a received linear combination. This is the case for the pattern in the right Table 3 (see also [17]). It is easy to see that, at time $t=\mu+1, \bar{E}_{1}^{t} \cap \bar{E}_{2}^{t}=\emptyset$, and additionally, $\bar{E}_{1}^{t} \subset \bar{E}_{4}^{t}, \bar{E}_{2}^{t} \subset \bar{E}_{4}^{t}$. To be rate optimal with respect to $r_{1}$ and $r_{2}$ we need, like before, to transmit from $\bar{E}_{1}^{t}+\bar{E}_{2}^{t}$. However, these transmissions cannot be decoded by $r_{4}$. Thus similarly to before we now have:

Proposition 5 If at time $t$ there exist receivers $r_{i}, r_{j}$ and $r_{k}$ such that $\bar{E}_{i}^{t} \cap \bar{E}_{j}^{t}=\emptyset, \bar{E}_{i}^{t} \subset \bar{E}_{k}^{t}, \bar{E}_{j}^{t} \subset \bar{E}_{k}^{t}$, and following time $t$, for the next $D$ timeslots that $r_{i}$ successfully receives so does $r_{j}$ and $r_{k}$, with $D \triangleq \min \left\{\left|\bar{E}_{i}^{t}\right|, \quad\left|\bar{E}_{j}^{t}\right|\right\}$, then offline coding results in delay $\mathcal{O}(D)$.

Clearly, coding allows to achieve delay one for a larger set than scheduling. Some additional such patterns are described in the following proposition (the proof can be found in the appendix).

Proposition 6 With coding we can achieve delay one when we have an arbitrary number of receivers $\rho$ and: 1. Erasure patterns where each broadcast transmission is successfully received by at most two receivers (this corresponds to high erasure probability). 2. Patterns where each broadcast transmission is not received by at most one receiver (this corresponds low erasure probability).

\subsection{Minimizing Delay with Coding Schemes is NP-hard}

Given that there exist instances where the problem becomes simpler with the use of coding, the next question is, whether the general problem, when we are allowed to use coding, becomes polynomial time, or remains $N P$-hard. Note that the problem of maximizing the throughput when multicasting over graphs becomes polynomial time if coding at intermediate network nodes is allowed [?, 21], while, if coding is not allowed, it is $N P$-hard. However the theorem below shows that this is not the case in our problem.

Theorem 2 Minimizing average and maximum delay in offline broadcasting in the presence of erasures and when the source uses (linear or nonlinear) coding is NP-hard.

The proof of this theorem appears in the Appendix due to space limitations and builds on the ideas in the proof of Theorem 1 .

We conclude this section with an inapproximability result following the definitions in [24] (the proof appears in the Appendix).

Proposition 7 Unless $P=N P$, there is no $\epsilon$-factor approximation (coding) algorithm for maximum delay in offline broadcasting with erasures for $\epsilon<1 / 2$. 


\section{Online Algorithms}

In this section, we discuss the competitive ratio of a natural class of systematic rate-optimal online algorithms for minimizing average (maximum) delay in the cases of arbitrary and i.i.d. erasures. We also suggest an online heuristic that improves significantly on the performance of the best heuristic from [17] for i.i.d. erasures.

A systematic coding algorithm uses the first $\mu$ transmissions to send all messages once uncoded and then starts sending combinations of messages. Rate optimal systematic algorithms have smaller average delay than their non-systematic variants where linear combinations of all messages are used for every transmission. The competitive ratio of systematic rate-optimal algorithms in the presence of a deterministic adversary who only knows that the source is using such an algorithm is given by the following proposition (a proof appears in the Appendix). The adversary is allowed to incur an erasure at any channel during any time step but is not allowed to eavesdrop any channel.

Proposition 8 For $\mu=O(\rho)$ and arbitrary erasures, a systematic rate-optimal online algorithm is $(\mu-O(1))$-competitive for minimizing average delay and $(\mu-1)$-competitive for minimizing maximum delay.

Proposition 8 motivates us to look at algorithms that are not rate-optimal in the online scenario. Specifically, we examine the case where all $\rho$ channels experience i.i.d. erasures with common constant erasure probability $q$. Figure 1 compares the performance of scheduling, two heuristics from [17], and a new heuristic algorithm that improves on the latter. A short description of these algorithms appears in the Appendix. As mentioned in Section 1, use of coding is critical to achieve lower delay as simple scheduling performs very poorly. The rate optimal algorithm (systematic FEC) achieves the expected delay of $\mu \cdot q$, which is significantly worse than the performance of our heuristic as $q, \rho$ (graphs below) and $\mu$ (simulations not shown here) increase. Hence the interdependence between delay from useless packets and delay from non instantly decodable packets has to be exploited in order to improve the performance of the online algorithm.
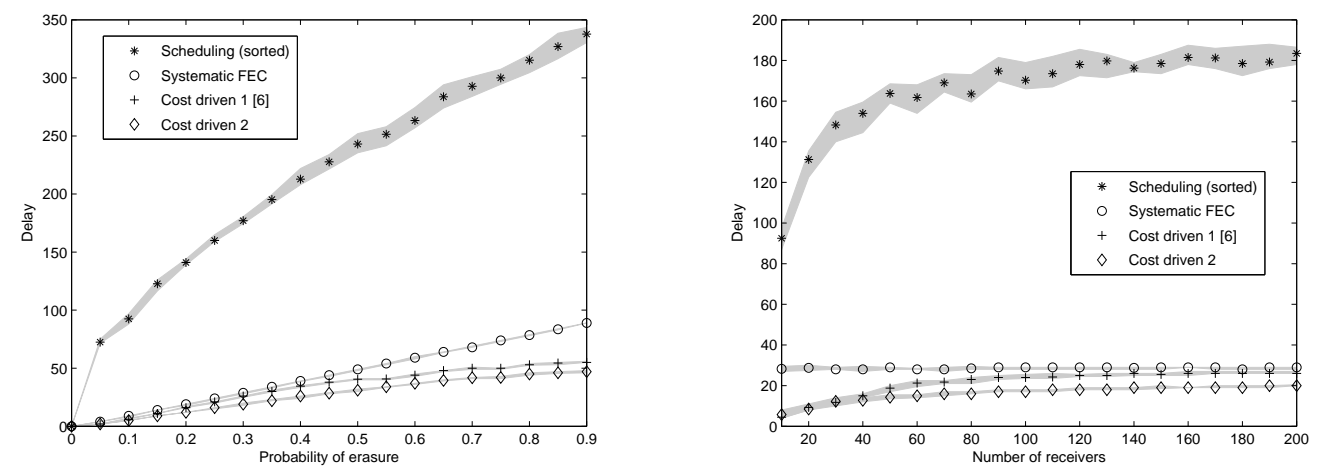

Figure 1: Median delay; the gray area corresponds to confidence interval; $\rho=150$ receivers on the left graph, erasure probability $q=0.3$ on the right graph; $\mu=100$ messages for both graphs

\section{Conclusions}

We address the question of finding the optimal offline algorithm for broadcast scheduling or coding that minimizes average and maximum delay when feedback is available. We show that the general problem is $N P$-hard both when the source uses scheduling and coding. However we show that coding is a reasonable alternative to scheduling as it can significantly reduce delay and alleviate the complexity of scheduling in certain instances. We also suggest online heuristics for minimizing average delay in the presence of i.i.d. erasures with the same erasure probability $q$ across receivers. 
The most important open questions at this point are the competitive analysis for more general online algorithms as well as the design of (additive) PTAS for the offline problem.

\section{References}

[1] R. Ahlswede, N. Cai, S-Y. R. Li, and R. W. Yeung, "Network information flow," IEEE Trans. on Information Theory, pp. 1204-1216, July 2000.

[2] N. Alon, A. Hassidim, E. Lubetzky, U. Stav, A. Weinstein, "Broadcasting with side information", FOCS 2008.

[3] J G. Apostolopoulos and M D. Trott. Path diversity for enhanced media streaming. IEEE Communications Magazine, 42(8): 80-87, August 2004.

[4] N. Bansal, D. Coppersmith, M. Sviridenko, "Improved approximation algorithms for broadcast scheduling", SODA 2006.

[5] Z. Bar-Yossef, Y. Birk, T. S. Jayram, T. Kol, "Index Coding with Side Information", FOCS 2006.

[6] Y. Birk, T. Kol, "Informed-source coding-on-demand (ISCOD) over broadcast channels, " Proc. IEEE INFOCOM, San Francisco, CA, 1998, pp. 1257-1264,1998.

[7] J. Chang, T. Erlebach, R. Gailis, S. Khuller, "Broadcast scheduling: algorithms and complexity", SODA 2008 , pages 473-482.

[8] M. Charikar, N. Bansal, S. Khanna, S. Naor, "Approximating the Average Response Time in Broadcast Scheduling", SODA 2005.

[9] S N. Diggavi, N.J.A. Sloane, and V. A. Vaishampayan. Asymmetric Multiple Description Lattice Vector Quantizers. IEEE Transactions on Information Theory, 48 (1): 174-191, January 2002.

[10] M. Durvy, C. Fragouli, P. Thiran, "On feedback for network coding", ISIT 2007.

[11] K. Foltz, L. Xu, J. Bruck, "Coding and Scheduling for Efficient Loss-Resilient Data Broadcasting", ISIT 2003.

[12] C. Fragouli and E. Soljanin, "Network coding: Fundamentals and Applications," Foundations and Trends in Networking, vol. 1, $2,2007$.

[13] C. Fragouli, D. Lun, M. Medard, P. Pakzad, "On feedback for network coding", Proceedings of CISS 2006.

[14] A. El Gamal and T. M. Cover, "Achievable rates for multiple descriptions," IEEE Trans. Information Theory, vol. 28, no. 6, pp. 851-857, Nov. 1982.

[15] V. K. Goyal, "Multiple Description Coding: Compression Meets the Network", IEEE Signal Processing Mag., vol. 18, no. 5, pp. 74-93, Sept. 2001.

[16] S. Katti, H. Rahul, W. Hu, D. Katabi, M. Médard, J. Crowcroft, "XORs in the Air: Practical Wireless Network Coding", Sigcomm06.

[17] L. Keller, E. Drinea, C. Fragouli, "Online broadcasting with Network Coding", Network Coding 2008.

[18] S-Y. R. Li, R. W. Yeung, and N. Cai, "Linear network coding," IEEE Trans. on Information Theory, vol. 49, Feb 2003.

[19] E. Lubetzky, U. Stav, "Non-Linear Index Coding Outperforming the Linear Optimum", FOCS 2007.

[20] M. Luby, "LT codes", IEEE Symposium on the Foundations of Computer Science (STOC), pp. 271-280, 2002.

[21] D. Lun, M. Médard, T. Ho, R. Koetter, "Network coding with a cost criterion", ISIT 2004.

[22] W. Mao, "Competitive analysis of on-line algorithms for on-demand data broadcast scheduling", ISPAN' 00.

[23] J. Massey, P. Massey, "Zero error with feedback", online tutorial.

[24] C. Papadimitriou, "Computational Complexity", Addison-Wesley, 1994.

[25] S. S. Pradhan, R. Puri, and K. Ramchandran, " $n$-channel symmetric multiple descriptions - Part I: $(n, k)$ source-channel erasure codes," IEEE Trans. Information Theory, vol. 50, pp. 47-61, Jan. 2004.

[26] R. Puri, S.S. Pradhan, and K. Ramchandran, " $n$-channel symmetric multiple descriptions - Part II: an achievable rate-distortion region," IEEE Trans. Information Theory, vol. 51, pp. 1377-1392, Apr. 2005.

[27] Sachs, D. G., Raghavan, A., and Ramchandran, K. 2000. Wireless Image Transmission Using Multiple-Description Based Concatenated Codes. In Proceedings of the Conference on Data Compression 2000, DCC.

[28] A. Shokrollahi, "Raptor Codes", IEEE Trans. Inf. Theory, vol. 52, 2006.

[29] C. Su, L. Tassiulas, V. J. Tsotras, "Broadcast scheduling for information distribution", Wireless Networks, Springer 2004.

[30] J. Sundararajan, D. Shah, M. Médard, "On queueing in coded networks -queue size follows degrees of freedom", ITW 2007. 


\section{Appendix}

\begin{tabular}{|c|c|c|c|c|c|c|c|c|c|c|}
\hline Time slot & $C_{1}^{1}$ & $C_{2}^{1}$ & $C_{1}^{2}$ & $C_{2}^{2}$ & $C_{1}^{3}$ & $C_{2}^{3}$ & $D^{1}$ & $D^{2}$ & $D^{3}$ & $D^{4}$ \\
\hline 1 & $\sqrt{ }$ & $\mathrm{X}$ & $\sqrt{ }$ & $\mathrm{X}$ & $\sqrt{ }$ & $\sqrt{ }$ & $\mathrm{X}$ & $\sqrt{ }$ & $\sqrt{ }$ & $\sqrt{ }$ \\
\hline 2 & $\mathrm{X}$ & $\mathrm{X}$ & $\mathrm{X}$ & $\mathrm{X}$ & $\sqrt{ }$ & $\sqrt{ }$ & $\mathrm{X}$ & $\sqrt{ }$ & $\sqrt{ }$ & $\sqrt{ }$ \\
\hline 3 & $\mathrm{X}$ & $\sqrt{ }$ & $\mathrm{X}$ & $\mathrm{X}$ & $\mathrm{X}$ & $\mathrm{X}$ & $\sqrt{ }$ & $\mathrm{x}$ & $\mathrm{X}$ & $\mathrm{X}$ \\
\hline 4 & $\mathrm{x}$ & $\mathrm{x}$ & $\mathrm{x}$ & $\sqrt{ }$ & $\mathrm{x}$ & $\mathrm{x}$ & $\sqrt{ }$ & $\mathrm{x}$ & $\mathrm{x}$ & $\mathrm{x}$ \\
\hline 5 & $\vec{V}$ & $\mathrm{X}$ & $\sqrt{ }$ & $\mathrm{x}$ & $\sqrt{ }$ & $\mathrm{X}$ & $\sqrt{ }$ & $\mathrm{X}$ & $\sqrt{ }$ & $\sqrt{ }$ \\
\hline 6 & $\mathrm{X}$ & $\mathrm{X}$ & $\mathrm{X}$ & $\mathrm{x}$ & $\mathrm{x}$ & $\mathrm{X}$ & $\sqrt{ }$ & $\mathrm{x}$ & $\sqrt{ }$ & $\sqrt{ }$ \\
\hline 7 & $\mathrm{X}$ & $\sqrt{ }$ & $\mathrm{X}$ & $\mathrm{X}$ & $\mathrm{X}$ & $\mathrm{X}$ & $\mathrm{X}$ & $\sqrt{ }$ & $\mathrm{X}$ & $\mathrm{X}$ \\
\hline 8 & $\mathrm{X}$ & $\mathrm{X}$ & $\mathrm{x}$ & $\sqrt{ }$ & $\mathrm{X}$ & $\sqrt{ }$ & $\mathrm{X}$ & $\sqrt{ }$ & $\mathrm{X}$ & $\mathrm{X}$ \\
\hline 9 & $\sqrt{ }$ & $\mathrm{X}$ & $\sqrt{ }$ & $\mathrm{X}$ & $\sqrt{ }$ & $\mathrm{x}$ & $\sqrt{ }$ & $\sqrt{ }$ & $\mathrm{x}$ & $\sqrt{ }$ \\
\hline 10 & $\mathrm{x}$ & $\mathrm{X}$ & $\mathrm{X}$ & $\mathrm{x}$ & $\mathrm{X}$ & $\mathrm{X}$ & $\sqrt{ }$ & $\sqrt{ }$ & $\mathrm{X}$ & $\sqrt{ }$ \\
\hline 11 & $\mathrm{X}$ & $\sqrt{ }$ & $\mathrm{x}$ & $\sqrt{ }$ & $\mathrm{X}$ & $\mathrm{X}$ & $\mathrm{X}$ & $\mathrm{x}$ & $\sqrt{ }$ & $\mathrm{x}$ \\
\hline 12 & $\mathrm{x}$ & $\mathrm{X}$ & $\mathrm{x}$ & $\mathrm{x}$ & $\mathrm{x}$ & $\sqrt{ }$ & $\mathrm{X}$ & $\mathrm{x}$ & $\sqrt{ }$ & $\mathrm{X}$ \\
\hline 13 & $\sqrt{ }$ & $\sqrt{ }$ & $\sqrt{ }$ & $\sqrt{ }$ & $\sqrt{ }$ & $\mathrm{X}$ & $\sqrt{ }$ & $\sqrt{ }$ & $\sqrt{ }$ & $\mathrm{x}$ \\
\hline 14 & $\sqrt{ }$ & $\sqrt{ }$ & $\sqrt{ }$ & $\sqrt{ }$ & $\mathrm{X}$ & $\mathrm{X}$ & $\sqrt{ }$ & $\sqrt{ }$ & $\sqrt{ }$ & $\mathrm{X}$ \\
\hline 15 & $\mathrm{X}$ & $\mathrm{X}$ & $\mathrm{X}$ & $\mathrm{X}$ & $\mathrm{X}$ & $\sqrt{ }$ & $\mathrm{X}$ & $\mathrm{x}$ & $\mathrm{X}$ & $\sqrt{ }$ \\
\hline 16 & $\mathrm{X}$ & $\mathrm{X}$ & $\mathrm{x}$ & $\mathrm{X}$ & $\mathrm{X}$ & $\mathrm{X}$ & $\mathrm{X}$ & $\mathrm{x}$ & $\mathrm{X}$ & $\sqrt{ }$ \\
\hline 17 & $\sqrt{ }$ & $\sqrt{ }$ & $\mathrm{x}$ & $\mathrm{X}$ & $\mathrm{x}$ & $\mathrm{X}$ & $\mathrm{X}$ & $\mathrm{x}$ & $\mathrm{X}$ & $\mathrm{X}$ \\
\hline 18 & $\mathrm{X}$ & $\sqrt{ }$ & $\mathrm{x}$ & $\mathrm{X}$ & $\mathrm{X}$ & $\mathrm{X}$ & $\mathrm{X}$ & $\mathrm{X}$ & $\mathrm{X}$ & $\mathrm{X}$ \\
\hline 19 & $\mathrm{X}$ & $\sqrt{ }$ & $\mathrm{x}$ & $\mathrm{X}$ & $\mathrm{X}$ & $\mathrm{X}$ & $\mathrm{X}$ & $\mathrm{X}$ & $\mathrm{X}$ & $\mathrm{X}$ \\
\hline 20 & $\sqrt{ }$ & $\mathrm{X}$ & $\mathrm{X}$ & $\mathrm{x}$ & $\mathrm{x}$ & $\mathrm{X}$ & $\mathrm{X}$ & $\mathrm{X}$ & $\mathrm{X}$ & $\mathrm{X}$ \\
\hline 21 & $\sqrt{ }$ & $\mathrm{X}$ & $\mathrm{x}$ & $\mathrm{X}$ & $\mathrm{X}$ & $\mathrm{X}$ & $\mathrm{X}$ & $\mathrm{x}$ & $\mathrm{X}$ & $\mathrm{X}$ \\
\hline 22 & $\mathrm{X}$ & $\mathrm{X}$ & $\sqrt{ }$ & $\sqrt{ }$ & $\mathrm{X}$ & $\mathrm{X}$ & $\mathrm{X}$ & $\mathrm{X}$ & $\mathrm{X}$ & $\mathrm{X}$ \\
\hline 23 & $\mathrm{X}$ & $\mathrm{X}$ & $\mathrm{X}$ & $\sqrt{ }$ & $\mathrm{X}$ & $\mathrm{X}$ & $\mathrm{X}$ & $\mathrm{x}$ & $\mathrm{X}$ & $\mathrm{X}$ \\
\hline 24 & $\mathrm{X}$ & $\mathrm{X}$ & $\mathrm{x}$ & $\sqrt{ }$ & $\mathrm{X}$ & $\mathrm{X}$ & $\mathrm{X}$ & $\mathrm{X}$ & $\mathrm{X}$ & $\mathrm{X}$ \\
\hline 25 & $\mathrm{X}$ & $\mathrm{X}$ & $\sqrt{ }$ & $\mathrm{X}$ & $\mathrm{x}$ & $\mathrm{X}$ & $\mathrm{X}$ & $\mathrm{X}$ & $\mathrm{X}$ & $\mathrm{X}$ \\
\hline 26 & $\mathrm{X}$ & $\mathrm{X}$ & $\sqrt{ }$ & $\mathrm{X}$ & $\mathrm{X}$ & $\mathrm{X}$ & $\mathrm{X}$ & $\mathrm{X}$ & $\mathrm{X}$ & $\mathrm{X}$ \\
\hline 27 & $\mathrm{X}$ & $\mathrm{X}$ & $\mathrm{x}$ & $\mathrm{X}$ & $\sqrt{ }$ & $\sqrt{ }$ & $\mathrm{X}$ & $\mathrm{X}$ & $\mathrm{X}$ & $\mathrm{X}$ \\
\hline 28 & $\mathrm{X}$ & $\mathrm{X}$ & $\mathrm{X}$ & $\mathrm{X}$ & $\mathrm{X}$ & $\sqrt{ }$ & $\mathrm{X}$ & $\mathrm{X}$ & $\mathrm{X}$ & $\mathrm{X}$ \\
\hline 29 & $\mathrm{X}$ & $\mathrm{X}$ & $\mathrm{x}$ & $\mathrm{X}$ & $\mathrm{X}$ & $\sqrt{ }$ & $\mathrm{X}$ & $\mathrm{X}$ & $\mathrm{X}$ & $\mathrm{X}$ \\
\hline 30 & $\mathrm{X}$ & $\mathrm{X}$ & $\mathrm{x}$ & $\mathrm{X}$ & $\sqrt{ }$ & $\mathrm{X}$ & $\mathrm{X}$ & $\mathrm{X}$ & $\mathrm{X}$ & $\mathrm{X}$ \\
\hline 31 & $\mathrm{X}$ & $\mathrm{X}$ & $\mathrm{x}$ & $\mathrm{X}$ & $\sqrt{ }$ & $\mathrm{X}$ & $\mathrm{X}$ & $\mathrm{X}$ & $\mathrm{X}$ & $\mathrm{X}$ \\
\hline
\end{tabular}

Table 4: $P(\phi)$ for the example formula $\phi=\left(x_{1} \vee x_{2} \vee x_{3}\right) \wedge\left(\bar{x}_{1} \vee \bar{x}_{2} \vee x_{3}\right) \wedge\left(\bar{x}_{2} \vee \bar{x}_{3} \vee x_{4}\right)$ and the scheduling construction in Section 3. 


\section{Minimizing Delay with Coding Schemes is NP-hard}

In this section we present the proof of Theorem 2 . Given an offline instance $B(m, n, h, P)$, the coding problem we are considering is to minimize the delay under any coding scheme that satisfies the instance. Again, delay here stands for average delay.

We will construct an offline broadcasting instance $B(\phi)=(\mu, \rho, \tau, P(\phi))$ such that $\phi$ is satisfiable if and only if there is a coding scheme that satisfies $B(\phi)$ with delay one. In our instance, the source has $\mu=3 n$ messages, there are $\rho=5 n+3 m$ receivers, and $\tau=10 n+6 m-3$ time slots. Our construction guarantees that each receiver has $\mu=3 n$ successful receptions within these $\tau$ time slots.

\begin{tabular}{|c|c|c|c|}
\hline Time slot & $C_{1}^{j}$ & $C_{2}^{j}$ & $C_{3}^{j}$ \\
\hline $5(i-1)+1$ & $\sqrt{ }$ & $\sqrt{ }$ & $\mathrm{x}$ \\
$5(i-1)+2$ & $\sqrt{ }$ & $\mathrm{x}$ & $\mathrm{x}$ \\
$5(i-1)+3$ & $\mathrm{x}$ & $\sqrt{ }$ & $\mathrm{x}$ \\
$5(i-1)+4$ & $\mathrm{x}$ & $\mathrm{x}$ & $\sqrt{ }$ \\
$5 i$ & $\mathrm{x}$ & $\mathrm{x}$ & $\mathrm{x}$ \\
\hline
\end{tabular}

\begin{tabular}{|c|c|c|c|}
\hline Time slot & $C_{1}^{j}$ & $C_{2}^{j}$ & $C_{3}^{j}$ \\
\hline $5(i-1)+1$ & $\sqrt{ }$ & $\sqrt{ }$ & $\mathrm{x}$ \\
$5(i-1)+2$ & $\sqrt{ }$ & $\mathrm{x}$ & $\mathrm{x}$ \\
$5(i-1)+3$ & $\mathrm{x}$ & $\sqrt{ }$ & $\mathrm{x}$ \\
$5(i-1)+4$ & $\mathrm{x}$ & $\mathrm{x}$ & $\mathrm{x}$ \\
$5 i$ & $\mathrm{x}$ & $\mathrm{x}$ & $\sqrt{ }$ \\
\hline
\end{tabular}

\begin{tabular}{|c|c|c|c|}
\hline Time slot & $C_{1}^{j}$ & $C_{2}^{j}$ & $C_{3}^{j}$ \\
\hline $5(i-1)+1$ & $\sqrt{ }$ & $\sqrt{ }$ & $\sqrt{ }$ \\
$5(i-1)+2$ & $\sqrt{ }$ & $\sqrt{ }$ & $\sqrt{ }$ \\
$5(i-1)+3$ & $\sqrt{ }$ & $\sqrt{ }$ & $\sqrt{ }$ \\
$5(i-1)+4$ & $\mathrm{x}$ & $\mathrm{x}$ & $\mathrm{x}$ \\
$5 i$ & $\mathrm{x}$ & $\mathrm{x}$ & $\mathrm{x}$ \\
\hline
\end{tabular}

Table 5: Erausure patterns for receivers $C_{1}^{j}, C_{2}^{j}, C_{3}^{j}$ during $\beta_{i}$. If clause $c_{j}$ contains $x_{i}$, they receive as in the left table; if $c_{j}$ contains $\bar{x}_{i}$, they receive as in the middle table; else $\left(c_{j}\right.$ does not contain $x_{i}$ or $\left.\bar{x}_{i}\right)$, they receive as in the right table.

\begin{tabular}{|c|c|c|c|c|c|}
\hline Time slot & $D_{1}^{\ell}$ & $D_{2}^{\ell}$ & $D_{3}^{\ell}$ & $D_{4}^{\ell}$ & $D_{5}^{\ell}$ \\
\hline $5(i-1)+1$ & $\sqrt{ }$ & $\sqrt{ }$ & $\mathrm{x}$ & $\sqrt{ }$ & $\sqrt{ }$ \\
$5(i-1)+2$ & $\sqrt{ }$ & $\sqrt{ }$ & $\mathrm{x}$ & $\sqrt{ }$ & $\sqrt{ }$ \\
$5(i-1)+3$ & $\sqrt{ }$ & $\sqrt{ }$ & $\sqrt{ }$ & $\sqrt{ }$ & $\sqrt{ }$ \\
$5(i-1)+4$ & $\mathrm{x}$ & $\mathrm{x}$ & $\sqrt{ }$ & $\mathrm{x}$ & $\mathrm{x}$ \\
$5 i$ & $\mathrm{x}$ & $\mathrm{x}$ & $\sqrt{ }$ & $\mathrm{x}$ & $\mathrm{x}$ \\
\hline
\end{tabular}

\begin{tabular}{|c|c|c|c|c|c|}
\hline Time slot & $D_{1}^{i}$ & $D_{2}^{i}$ & $D_{3}^{i}$ & $D_{4}^{i}$ & $D_{5}^{\imath}$ \\
\hline $5(i-1)+1$ & $\sqrt{ }$ & $\mathrm{x}$ & $\mathrm{x}$ & $\mathrm{x}$ & $\mathrm{x}$ \\
$5(i-1)+2$ & $\sqrt{ }$ & $\sqrt{ }$ & $\mathrm{x}$ & $\mathrm{x}$ & $\mathrm{x}$ \\
$5(i-1)+3$ & $\sqrt{ }$ & $\sqrt{ }$ & $\sqrt{ }$ & $\mathrm{x}$ & $\mathrm{x}$ \\
$5(i-1)+4$ & $\mathrm{x}$ & $\mathrm{x}$ & $\sqrt{ }$ & $\sqrt{ }$ & $\mathrm{x}$ \\
$5 i$ & $\mathrm{x}$ & $\mathrm{x}$ & $\sqrt{ }$ & $\sqrt{ }$ & $\sqrt{ }$ \\
\hline
\end{tabular}

Table 6: Let $1 \leq k \leq 5$. During $\beta_{i}, D_{k}^{\ell}$ receive as shown in the left table if $\ell<i$; the right table shows receptions for $D_{k}^{i}$.

Our construction works as follows. For every variable $x_{i}$, we introduce 3 messages, $e_{i}, \bar{e}_{i}$ and $e_{i}^{\prime}$. For every clause $c_{j}, 1 \leq j \leq m$, we introduce three receivers, denoted by $C_{1}^{j}, C_{2}^{j}$ and $C_{3}^{j}$. For every variable $x_{i}, 1 \leq i \leq n$, we introduce five receivers, denoted by $D_{k}^{i}$, for $1 \leq k \leq 5$, whose role will be discussed after completing the construction of the erasure pattern.

Also, for every variable $x_{i}$, we introduce 5 consecutive time slots, which we call the variable period $\beta_{i} ; \beta_{i}$ starts at time slot $5(i-1)+1$, and ends at time slot $5 i$. Following the $n$-th variable period, we introduce $m$ consecutive clause periods: the $j$-th clause period, denoted by $\gamma_{j}$, consists of 6 time slots, starts at time slot $5 n+6(j-1)+1$, and ends at time slot $5 n+6 j$. Finally, following the $m$ clause periods, we introduce $5 n-3$ time slots, which we call "patching" time slots, because their role is simply to provide sufficient time for the receivers $D_{k}^{i}$ to obtain all messages.

We now proceed to giving values to the $\tau \cdot \rho$ entries of the erasure matrix $P$. We will do this sequentially in time, i.e., by first considering the variable periods, then the clause periods, and finally the "patching" time slots.

During variable period $\beta_{i}$, for all $1 \leq j \leq n$, receivers $C_{1}^{j}, C_{2}^{j}, C_{3}^{j}$ corresponding to clause $c_{j}$ receive as shown in Table 5 depending on whether $x_{i}, \bar{x}_{i}$ or none of them appears in $c_{j}$. Also, during $\beta_{i}$, receivers $D_{k}^{\ell}$ with $1 \leq k \leq 5$ receive as shown in Table 6 if $\ell \leq i$; otherwise (if $\ell>i$ ), they all experience erasures.

During clause period $\gamma_{j}$, receivers $C_{1}^{j}, C_{2}^{j}, C_{3}^{j}$ corresponding to clause $c_{j}$ receive as shown in Table 7 . All other receivers experience erasures during $\gamma_{j}$.

Finally, consider the "patching" time slots. We can think of them as being grouped into $n-1$ periods of 5 time slots, and one last period of only two time slots. For $1 \leq i \leq n$, at time $5 n+6 m+5(i-1)+1$, receivers $D_{4}^{i}, D_{5}^{i}$ receive. At the next time slot $5 n+6 m+5(i-1)+2$, receivers $D_{2}^{i}, D_{5}^{i}$ receive. For $1 \leq i \leq n-1$, during the last three time slots of patching period $i$, all receivers $D_{k}^{\ell}$ with $\ell>i, 1 \leq k \leq 5$ receive.

The above completes our construction. Table 8 shows $P(\phi)$ for the example formula $\phi=\left(x_{1} \vee x_{2} \vee x_{3}\right) \wedge\left(\bar{x}_{1} \vee \bar{x}_{2} \vee x_{3}\right) \wedge\left(\bar{x}_{2} \vee \bar{x}_{3} \vee x_{4}\right)$ for which $B(\phi)=(12,29,55, P(\phi))$.

Again it is easy to check that the reduction can be carried out by a deterministic Turing machine in logarithmic space and that all receivers have exactly $3 n$ successful receptions by time $\tau$, hence any rate-optimal scheme would satisfy $B(\phi)$. Here the role of the receivers $D_{k}^{i}$ is twofold (see Lemma 1 for a proof): they guarantee that (a) a single message is scheduled during every time slot of every $\beta_{i}$, and (b) exactly 3 messages are sent during each $\beta_{i}$, with the two messages sent during the first two time slots being rescheduled during the last two time slots, in any order. Similarly to Section 3, this flexibility in the scheduling of the messages during the last two time slots of each $\beta_{i}$ is our choice gadget. Our consistency gadget is that during $\beta_{i}, C_{3}^{j}$ receives a different message from $C_{3}^{\ell}$ when $x_{i}$ appears in clause $c_{j}$ and $\bar{x}_{i}$ in $c_{\ell}$. Finally our clause constraint gadget is the simultaneous reception of the three receivers corresponding to clause $c_{j}$ during the first time slot of $\gamma_{j}$.

We now move to showing that $\phi$ is satisfiable if and only if $B(\phi)$ admits delay one. Before, we introduce the following two schedulings that will prove useful for our arguments.

Scheduling 1 for $\beta_{i}$ : the ordered sequence of messages $e_{i}, \bar{e}_{i}, e_{i}^{\prime}, \bar{e}_{i}, e_{i}$. 


\begin{tabular}{|c|c|c|c|}
\hline Time slot & $C_{1}^{j}$ & $C_{2}^{j}$ & $C_{3}^{j}$ \\
\hline $5 n+6(j-1)+1$ & $\sqrt{ }$ & $\sqrt{ }$ & $\sqrt{ }$ \\
$5 n+6(j-1)+2$ & $\mathrm{x}$ & $\mathrm{x}$ & $\sqrt{ }$ \\
$5 n+6(j-1)+3$ & $\mathrm{x}$ & $\sqrt{ }$ & $\sqrt{ }$ \\
$5 n+6(j-1)+4$ & $\sqrt{ }$ & $\mathrm{x}$ & $\sqrt{ }$ \\
$5 n+6(j-1)+5$ & $\mathrm{x}$ & $\sqrt{ }$ & $\sqrt{ }$ \\
$5 n+6 j$ & $\sqrt{ }$ & $\mathrm{x}$ & $\sqrt{ }$ \\
\hline
\end{tabular}

Table 7: Receiving pattern of $C_{1}^{j}, C_{2}^{j}, C_{3}^{j}$ during clause period $\gamma_{j}$.

Scheduling 2 for $\beta_{i}$ : the ordered sequence of messages $e_{i}, \bar{e}_{i}, e_{i}^{\prime}, e_{i}, \bar{e}_{i}$.

Proposition 9 If $\phi$ is satisfiable, then there is a coding scheme $T$ that satisfies the offline broadcasting instance $B(\phi)=(3 n, 3 m+$ $5 n, 10 n+6 m-3, P(\phi))$ with delay one.

Proof. Consider a satisfying truth assignment for $\phi$. For $1 \leq i \leq n$, if $x_{i}$ is true, the coding scheme $T$ applies Scheduling 1 for $\beta_{i}$ during variable period $\beta_{i}$. Othewise, if $x_{i}$ is false, it applies Scheduling 2 for $\beta_{i}$ during $\beta_{i}$. Then the first $3 n$ transmissions of $T$ incur delay one, and $D_{1}^{1}, D_{3}^{1}$ obtain all messages.

Since $\phi$ is satisfiable, every clause has at least one literal that is true. W.l.o.g., let $c_{j}=\left(\ell_{i} \vee \ell_{a} \vee \ell_{b}\right)$ be any clause, where $\ell_{y}$ is either $x_{y}$ or $\bar{x}_{y}$, and suppose that $\ell_{i}$ is (one of) the satisfying literal(s) for this clause, i.e., $x_{i}$ is set to true if and only $\ell_{i}=x_{i}$. We now show how $T$ completes the clause periods so that the clause receivers obtain all messages without delay.

By time $5 n$, receivers $C_{1}^{j}, C_{2}^{j}, C_{3}^{j}$ know $3 n-9$ messages, i.e., all messages corresponding to the variables that do not appear in clause $c_{j}$. Further (see Table 5), $C_{1}^{j}$ knows $\left\{e_{i}, \bar{e}_{i}, e_{a}, \bar{e}_{a}, e_{b}, \bar{e}_{b}\right\}, C_{2}^{j}$ knows $\left\{e_{i}, e_{i}^{\prime}, e_{a}, e_{a}^{\prime}, e_{b}, e_{b}^{\prime}\right\}$, and $C_{3}^{j}$ knows exactly one from $\left\{e_{a}, \bar{e}_{a}\right\}$ and one from $\left\{e_{b}, \bar{e}_{b}\right\} . C_{3}^{j}$ also knows $\bar{e}_{i}$ which he received at the fourth time slot of $\beta_{i}$ if $\ell_{i}=x_{i}$ (in which case, $x_{i}$ was set to true, and $T$ applied Scheduling 1 for $\beta_{i}$ during $\beta_{i}$ ), or at the fifth time slot of $\beta_{i}$ if $\ell_{i}=\bar{x}_{i}$ (in which case, $x_{i}$ was set to false and $T$ applied Scheduling 2).

During $\gamma_{j}$ (see Table 7), $T$ sends the following packets: first it sends $\bar{e}_{i}+e_{i}^{\prime}$. This results in $C_{1}^{j}$ obtaining $e_{i}^{\prime}, C_{2}^{j}$ obtaining $\bar{e}_{i}$, and $C_{3}^{j}$ obtaining $e_{i}^{\prime}$. Next it sends $e_{i}$. During the third and the fourth slots, if $C_{3}^{j}$ received $e_{a}$ during $\beta_{a}$, it sends $e_{a}+\bar{e}_{a}$, and $e_{a}^{\prime}$ respectively. Otherwise, if $C_{3}^{j}$ received $\bar{e}_{a}$ during $\beta_{a}$, it sends $\bar{e}_{a}+e_{a}^{\prime}$ and $e_{a}+e_{a}^{\prime}$ respectively. The situation for $\ell_{b}$ is identical. Hence by time $5 n+6 j, C_{1}^{j}, C_{2}^{j}$ and $C_{3}^{j}$ have obtained all messages without delay.

At time $5 n+6 m+1$, every receiver $D_{k}^{i}$ except for $D_{1}^{1}$ and $D_{3}^{1}$ still needs some messages. For $1 \leq i \leq n$, at the first time slot of the $i$-th patching period, $T$ sends $e_{i}^{\prime}$, which both $D_{4}^{i}$ and $D_{5}^{i}$ need (see Table 6). At the second slot, it sends $e_{i}+\bar{e}_{i}$ if $x_{i}$ was set to true, or $e_{i}$ if $x_{i}$ was set to false; this satisfies both $D_{2}^{i}$ and $D_{5}^{i}$. At this point, $D_{2}^{i}, D_{4}^{i}, D_{5}^{i}$ have received all messages. For $1 \leq i \leq n-1$ 5 , during the last three slots of patching period $i, T$ sends $e_{i}, \bar{e}_{i}, e_{i}^{\prime}$, which receivers $D_{k}^{\ell}$ with $\ell>i$ had missed during $\beta_{i}$. These transmissions result in $D_{1}^{i+1}, D_{3}^{i+1}$ obtaining all messages. This explains why the $n$-th patching period consists of only two slots: the only receivers still requiring some messages are $D_{2}^{n}, D_{4}^{n}, D_{5}^{n}$. We conclude that by the end of the $n$-th patching period all receivers know all messages. Hence $T$ satisfies $B(\phi)$ with delay one.

Conversely, consider any coding scheme $T^{\prime}$ that satisfies $B(\phi)$ with delay one. We will exhibit a satisfying truth assignment for $\phi$. We first extend our previous notation of $E_{i}^{t_{1} \ldots t_{T}}$ to include all time steps $1 \leq t_{1} \leq t_{T} \leq 5$. We now show a technical but useful lemma concerning properties of $T^{\prime}$ during the variable periods.

Lemma 1 Any coding scheme $T^{\prime}$ that satisfies $B(\phi)$ with delay one is rate-optimal and sends a single message at every time step $j$ for $1 \leq j \leq 5 n$. Moreover $T^{\prime}$ sends exactly 3 messages during every $\beta_{i}$, with the messages sent during the first two time slots of $\beta_{i}$ being resent (in some order) during the last two time slots of $\beta_{i}$. After $\beta_{i}$, these messages will not be sent again before (potentially) time $5 n+1$.

Proof. Trivially, $T^{\prime}$ must be rate-optimal. Suppose that at time step $5(i-1)+k$, for any $1 \leq i \leq n$ and $1 \leq k \leq 5$, a (linear or nonlinear) function of at least two messages is sent. Then receiver $D_{k}^{i}$ will delay, as he has received nothing so far.

For the second part of the lemma, we need to show that $E_{i}^{12345^{k}} \cap E_{j}^{12345}=\emptyset$ for all $1 \leq i \neq j \leq n$. Also, we must show that $E_{i}^{12}=E_{i}^{45}$

Since $D_{1}^{1}$ receives during the first three time slots of every $\beta_{i}$, and the scheme is rate-optimal, $E_{i}^{123} \cap E_{j}^{123}=\emptyset$ for all $1 \leq i<j \leq n$. Similarly, since $D_{3}^{1}$ receives during the last three time slots of all $\beta_{i}$, and $T^{\prime}$ is rate-optimal, $E_{i}^{345} \cap E_{j}^{345}=\emptyset$ for all $1 \leq i<j \leq n$. Therefore we need show that $E_{i}^{12} \cap E_{j}^{45}=\emptyset$ for all $1 \leq i \neq j \leq n$. Equivalently we just need to show that $E_{i}^{12}=E_{i}^{45}$.

Oberve that, for all $i, D_{4}^{i}$ receives $E_{i}^{45} \cup E_{i+1}^{123} \cup \ldots \cup E_{n}^{123}$, and $T^{\prime}$ is rate-optimal. Hence $E_{i}^{45} \cap E_{j}^{123}=\emptyset$ for all $j>i$.

Moreover, for example for $i=1,\left|E_{1}^{45} \cup E_{2}^{123} \cup \ldots \cup E_{n}^{123}\right|=3 n-1$. Hence $D_{4}^{1}$ knows $3 n-1$ messages by time $5 n$. Since $T^{\prime}$ satisfies $B(\phi)$ and $D_{1}^{1}$ does not receive after time $5 n, D_{1}^{1}$ must know all $3 n$ messages by time $5 n$. Since $D_{1}^{1}$ and $D_{4}^{1}$ know the same $3 n-3$ messages corresponding to $E_{2}^{123} \cup \ldots \cup E_{n}^{123}$, it follows that $E_{1}^{45} \subset E_{1}^{123}$. Since $E_{1}^{3} \not \subset E_{1}^{12}$ as $D_{1}^{1}$ receives during all three time steps $1,2,3$, and $E_{1}^{3} \not \subset E_{1}^{45}$ as $D_{3}^{1}$ receives during all three time steps $3,4,5$, we conclude that $E_{1}^{12}=E_{1}^{45}$. The same argument holds for all $i>1$.

\footnotetext{
${ }^{5}$ Recall that the last patching period consists of only two time slots.
} 
W.l.o.g., assume that $T^{\prime}$ schedules the three messages $e_{x_{i}}, e_{y_{i}}, e_{z_{i}}$ during the first three time slots of $\beta_{i}$, in this order. By Lemma 1 , these messages will not be rescheduled before time $5 n$, so for the sake of clarity, we may relabel them as $e_{i}, \bar{e}_{i}, e_{i}^{\prime}$ respectively. We define the following truth assignment. For $1 \leq i \leq n$, if $T^{\prime}$ applied Scheduling 1 for $\beta_{i}$ during $\beta_{i}, x_{i}$ is set to true, else if $T^{\prime}$ used Scheduling 2 for $\beta_{i}$ during $\beta_{i}, x_{i}$ is set to false. Notice that Lemma 1 guarantees that any $T^{\prime}$ applied one of these two schedulings indeed during $\beta_{i}$.

Similarly to Lemma 1, the following proposition presents necessary and sufficient conditions for the clause periods to be completed with delay one.

Proposition 10 Let $c_{j}=\left(\ell_{i} \vee \ell_{a} \vee \ell_{b}\right)$ be any clause. In any scheme $T^{\prime}$ that satisfies $B(\phi)$ with delay one, $C_{3}^{j}$ has received at least one of $\bar{e}_{i}, \bar{e}_{a}, \bar{e}_{b}$ by time $5 n$.

Before we give the proof of Proposition 10, we show how it concludes the second direction of our reduction.

Corollary 2 If $T^{\prime}$ is a coding scheme that satisfies $B(\phi)$ with delay one then $\phi$ is satisfiable.

Proof. Consider any clause $c_{j}=\left(\ell_{i} \vee \ell_{a} \vee \ell_{b}\right)$. By Proposition 10, $T^{\prime}$ is such that $C_{3}^{j}$ has received at least one of $\bar{e}_{i}, \bar{e}_{a}, \bar{e}_{b}$ by time $5 n$. W.l.o.g., assume $C_{3}^{j}$ received $\bar{e}_{i}$. If $C_{3}^{j}$ received this message at time $5(i-1)+4$, then $x_{i}$ appears in $c_{j}$ and $T^{\prime}$ used Scheduling 1 for $\beta_{i}$. Hence our truth assignement set $x_{i}$ to true. Otherwise, if $C_{3}^{j}$ received $\bar{e}_{i}$ at time $5 i$, then $\bar{x}_{i}$ appears in $c_{j}$ and $T^{\prime}$ used Scheduling 2 for $\beta_{i}$. Hence our truth assignement set $x_{i}$ to false. In either case, our truth assignment for $x_{i}$ satisfies $c_{j}$. Since Proposition 10 applies to all $C_{3}^{j}$ for $1 \leq j \leq m$, there is (at least) one literal for every clause that is set to true by our truth assignment. Hence $\phi$ is satisfiable.

We now give the proof of Proposition 10

Proof. Consider the 3 receivers $C_{1}^{j}, C_{2}^{j}, C_{3}^{j}$ corresponding to $c_{j}$. By Lemma 1 , under any $T^{\prime}$, at the beginning of $\gamma_{j}$, each of $C_{1}^{j}, C_{2}^{j}, C_{3}^{j}$ knows the $3 n-9$ messages that correspond to the $n-3$ variables that do not appear in $c_{j}$. Further, $C_{1}^{j}$ knows $\left\{e_{i}, \bar{e}_{i}, e_{a}, \bar{e}_{a}, e_{b}, \bar{e}_{b}\right\}$, $C_{2}^{j}$ knows $\left\{e_{i}, e_{i}^{\prime}, e_{a}, e_{a}^{\prime}, e_{b}, e_{b}^{\prime}\right\}$ and $C_{3}^{j}$ knows exactly one of $e_{i}, \bar{e}_{i}$, one of $e_{a}, \bar{e}_{a}$, and one of $e_{b}, \bar{e}_{b}$.

Suppose that $C_{3}^{j}$ did not receive any of $\bar{e}_{i}, \bar{e}_{a}, \bar{e}_{b}$. Then he received $e_{i}, e_{a}, e_{b}$. Now at the first slot of $\gamma_{j}$ where $C_{1}^{j}, C_{2}^{j}, C_{3}^{j}$ receive simultaneously, there is no way to avoid delay: $C_{3}^{j}$ needs exactly one single message either from $\left\{\bar{e}_{i}, \bar{e}_{a}, \bar{e}_{b}\right\}$, or one from $\left\{e_{i}^{\prime}, e_{a}^{\prime}, e_{b}^{\prime}\right\}$, while $C_{1}^{j}$ needs no single message from the first set and $C_{2}^{j}$ needs no single message from the second set.

On the other hand suppose that $C_{3}^{j}$ received at least one of $\bar{e}_{i}, \bar{e}_{a}, \bar{e}_{b}$, w.l.o.g. $\bar{e}_{i}$. Then at time $5 n+6(j-1)+1$, a function of $\bar{e}_{i}$ and exactly one from $\left\{e_{i}^{\prime}, e_{a}^{\prime}, e_{b}^{\prime}\right\}$ would suffice to incur zero delay to all 3 receivers. 
Proposition 6 With coding we can achieve delay one when we have an arbitrary number of receivers $\rho$ and: 1 . Erasure patterns where each broadcast transmission is successfully received by at most two receivers (this corresponds to high erasure probability). 2. Patterns where each broadcast transmission is not received by at most one receiver (this corresponds low erasure probability).

Proof. For two sets $A, B$, the notation $A+B$ means $\{a+b: a \in A, b \in B\}$.

1. If two receivers $r_{i}$ and $r_{j}$ receive, transmit a message from $\bar{E}_{i}^{t} \cap \bar{E}_{j}^{t}$ if not empty, otherwise from $\bar{E}_{i}^{t}+\bar{E}_{j}^{t}$ if not empty, otherwise from $\bar{B}_{t}$. If only $r_{i}$ receives, if $\bar{E}_{i}^{t} \neq \emptyset$ transmit from $\bar{E}_{i}^{t}$ otherwise transmit a message from $\bar{E}_{i}^{t}$, otherwise a message from $\bar{B}_{t}$.

2. Use systematic transmission for $\mu$ time slots. After $\mu$ timeslots there exists at most one receiver who has not received each message. Continue by transmitting messages from $+{ }_{i} \bar{E}_{i}^{t}$, where in the summation we include all receivers $i$ that receive the transmission (and have not completed reception).

Proposition 7 Unless $P=N P$, there is no $\epsilon$-factor approximation (coding) algorithm for maximum delay in offline broadcasting with erasures for $\epsilon<1 / 2$.

Proof. Let $O P T$ be the optimal algorithm for offline broadcasting in the presence of erasures, and $O P T(x)$ the maximum delay incurred by $O P T$ on instance $x=(\mu, \rho, \tau, P)$. Let $T_{C}$ be an $\epsilon$-factor approximation algorithm for maximum delay in offline broadcasting with erasures for some $\epsilon<1 / 2$. Then for all inputs $x$ to $T_{C}, T_{C}(x) \leq \frac{1}{1-\epsilon} \cdot O P T(x)$.

Given a formula $\phi$ in $3 \mathrm{CNF}$, we construct $B(\phi)$ in polynomial time as described in the proof of Theorem 2 and run $T_{C}$ on input $B(\phi)$. There are two cases: if $T_{C}(B(\phi))=1$, then we know that $\phi$ is satisfiable. Otherwise, if $T_{C}(B(\phi)) \geq 2$, then we conclude that

$$
2 \leq T_{C}(B(\phi)) \leq \frac{1}{1-\epsilon} \cdot O P T(B(\phi)) \Rightarrow 2(1-\epsilon) \leq O P T(B(\phi)) \Rightarrow 1<O P T(B(\phi))
$$

Since the maximum delay of $B(\phi)$ is one if and only if $\phi$ is not satisfiable, we conclude that $\phi$ is not satisfiable. Since $B(\phi)$ can be constructed in polynomial time, and $T_{C}$ runs in polynomial time, we can decide whether $\phi$ is satisfiable in polynomial time.

Proposition 8 For $\mu=O(\rho)$ and arbitrary erasures, a systematic rate-optimal online algorithm is ( $\mu$ - O(1))-competitive for average delay and $(\mu-1)$-competitive for maximum delay.

Proof. During the first $\mu$ transmissions, the adversary allows receiver $r_{j}$ to obtain transmission $j$ and causes erasures to all other receivers. After the $\mu$-th transmission the adversary allows all $\rho$ receivers to successfully receive all transmitted packets.

Since exactly one receiver has obtained each message by time $\mu$, the source must transmit linear combinations of all messages starting at time $\mu+1$ in order to be rate optimal for every receiver. This incurs a total delay of

$$
\rho+(\mu-2) \cdot \rho+(\rho-\mu)
$$

during the following $\mu$ transmissions. The first term comes from $t=0$, the second term from time $\mu+1$ up to time $2 \mu-2$ where all receivers delay, and the last term from time $2 \mu-1$ where receivers $r_{\mu+1}, \ldots, r_{\rho}$ delay (at this point, receivers $r_{1}, \ldots, r_{\mu}$ have successfully received $\mu$ packets hence can decode for the $\mu$ messages and do not delay). It follows that the average delay is given by

$$
\mu-\frac{\mu}{\rho}=\mu-O(1)
$$

while the maximum delay is $\mu-1$ (the delay of any receiver $r_{j}$ with $j>\mu$ ).

On the other hand, the optimal offline algorithm incurs average and maximum delay of one: it only transmits $e_{1}$ during the first $\mu$ transmissions, followed by $e_{2}, \ldots, e_{\mu}, e_{1}$ during the following $\mu$ transmissions. The proposition follows.

\section{Short Description of the Heuristics in Figure 1, Section 5}

1. Scheduling (sorted): At every time slot, the message that is requested by most receivers is transmitted.

2. Systematic FEC [17]: During the first $\mu$ transmissions, all $\mu$ messages are transmitted once. Then packets with random linear combinations of the messages are transmitted so that every transmitted packet is rate optimal for every receiver.

3. Cost driven 2: This is a variant of Cost driven 1 from [17]. During the first $\mu$ transmissions, all $\mu$ messages are transmitted once. From then on, the packet $p(t)$ is built as follows: first a packet $s(t)$ that is instantly decodable for every receiver is built in a random manner; $s(t)$ might not be novel for every receiver. At this point, the algorithm sets $p(t)=s(t)$ and then augments $p(t)$ by adding more messages to build the final packet as follows. While there are still messages that are not included in $p(t)$ and have not yet been chosen by the algorithm, one such message $m$ is chosen at random. This message is added to $p(t)$ (multiplied by a random coefficient $c$ ) according to the following condition. Assume that all receivers will receive $p(t)$. If the number of receivers that will not experience delay upon reception of $p(t)+c \cdot m$ is larger than the number of receivers that will not experience delay upon reception of $p(t)$, then set $p(t)=p(t)+c \cdot m$. When the erasures are i.i.d. and identically distributed across receivers, the assumption that all receivers will receive $p(t)$ may be replaced by the assumption that the expected number of receivers receive $p(t)$ without affecting the condition for adding $m$ to $p(t)$. 


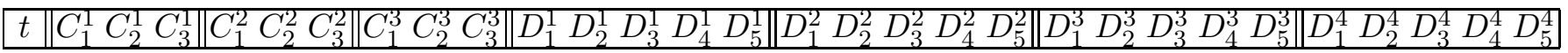
\begin{tabular}{|c||ccc||ccc||ccc||ccccc||ccccc||ccccc||ccccc|}
\hline \hline 1 & $\sqrt{ }$ & $\sqrt{ }$ & $\mathrm{x}$ & $\sqrt{ }$ & $\sqrt{ }$ & $\mathrm{x}$ & $\sqrt{ }$ & $\sqrt{ }$ & $\sqrt{ }$ & $\sqrt{ }$ & $\mathrm{x}$ & $\mathrm{x}$ & $\mathrm{x}$ & $\mathrm{x}$ & $\mathrm{x}$ & $\mathrm{x}$ & $\mathrm{x}$ & $\mathrm{x}$ & $\mathrm{x}$ & $\mathrm{x}$ & $\mathrm{x}$ & $\mathrm{x}$ & $\mathrm{x}$ & $\mathrm{x}$ & $\mathrm{x}$ & $\mathrm{x}$ & $\mathrm{x}$ & $\mathrm{x}$ & $\mathrm{x}$ \\
\hline
\end{tabular}

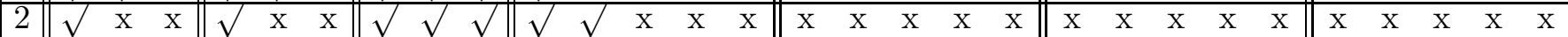
\begin{tabular}{|l||cc|c||ccc||ccc||cccccc||ccccc||ccccc||ccccc|}
\hline 3 & $\mathrm{x}$ & $\sqrt{ }$ & $\mathrm{x}$ & $\mathrm{x}$ & $\sqrt{ }$ & $\mathrm{x}$ & $\sqrt{ }$ & $\sqrt{ }$ & $\sqrt{ }$ & $\sqrt{ }$ & $\sqrt{ }$ & $\sqrt{ }$ & $\mathrm{x}$ & $\mathrm{x}$ & $\mathrm{x}$ & $\mathrm{x}$ & $\mathrm{x}$ & $\mathrm{x}$ & $\mathrm{x}$ & $\mathrm{x}$ & $\mathrm{x}$ & $\mathrm{x}$ & $\mathrm{x}$ & $\mathrm{x}$ & $\mathrm{x}$ & $\mathrm{x}$ & $\mathrm{x}$ & $\mathrm{x}$ & $\mathrm{x}$ \\
\hline
\end{tabular} \begin{tabular}{|l||ccc||ccc||ccc||cccccc||ccccc||ccccc||ccccc|}
\hline 4 & $\mathrm{x}$ & $\mathrm{x}$ & $\sqrt{ }$ & $\mathrm{x}$ & $\mathrm{x}$ & $\mathrm{x}$ & $\mathrm{x}$ & $\mathrm{x}$ & $\mathrm{x}$ & $\mathrm{x}$ & $\mathrm{x}$ & $\sqrt{ }$ & $\sqrt{ }$ & $\mathrm{x}$ & $\mathrm{x}$ & $\mathrm{x}$ & $\mathrm{x}$ & $\mathrm{x}$ & $\mathrm{x}$ & $\mathrm{x}$ & $\mathrm{x}$ & $\mathrm{x}$ & $\mathrm{x}$ & $\mathrm{x}$ & $\mathrm{x}$ & $\mathrm{x}$ & $\mathrm{x}$ & $\mathrm{x}$ & $\mathrm{x}$ \\
\hline
\end{tabular} \begin{tabular}{|l|lll||lll||lll||llllll||llllll||lllll||lllll|}
\hline 5 & $\mathrm{x}$ & $\mathrm{x}$ & $\mathrm{x}$ & $\mathrm{x}$ & $\mathrm{x}$ & $\sqrt{ }$ & $\mathrm{x}$ & $\mathrm{x}$ & $\mathrm{x}$ & $\mathrm{x}$ & $\mathrm{x}$ & $\sqrt{ }$ & $\sqrt{ }$ & $\sqrt{ }$ & $\mathrm{x}$ & $\mathrm{x}$ & $\mathrm{x}$ & $\mathrm{x}$ & $\mathrm{x}$ & $\mathrm{x}$ & $\mathrm{x}$ & $\mathrm{x}$ & $\mathrm{x}$ & $\mathrm{x}$ & $\mathrm{x}$ & $\mathrm{x}$ & $\mathrm{x}$ & $\mathrm{x}$ & $\mathrm{x}$ \\
\hline
\end{tabular}

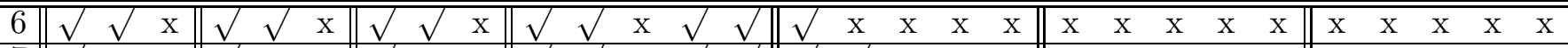

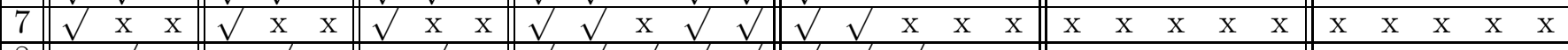

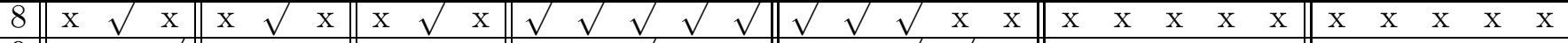

\begin{tabular}{||c|cc||ccc||ccc||cccccc||ccc|c|c||ccccc||ccccc|}
\hline 9 & $\mathrm{x}$ & $\mathrm{x}$ & $\sqrt{ }$ & $\mathrm{x}$ & $\mathrm{x}$ & $\mathrm{x}$ & $\mathrm{x}$ & $\mathrm{x}$ & $\mathrm{x}$ & $\mathrm{x}$ & $\mathrm{x}$ & $\sqrt{ }$ & $\mathrm{x}$ & $\mathrm{x}$ & $\mathrm{x}$ & $\mathrm{x}$ & $\sqrt{ }$ & $\sqrt{ }$ & $\mathrm{x}$ & $\mathrm{x}$ & $\mathrm{x}$ & $\mathrm{x}$ & $\mathrm{x}$ & $\mathrm{x}$ & $\mathrm{x}$ & $\mathrm{x}$ & $\mathrm{x}$ & $\mathrm{x}$ & $\mathrm{x}$ \\
\hline
\end{tabular}

\begin{tabular}{|l||lll||lll||lll||llllll||lllllll|llllll||lllll|}
\hline 10 & $\mathrm{x}$ & $\mathrm{x}$ & $\mathrm{x}$ & $\mathrm{x}$ & $\mathrm{x}$ & $\sqrt{ }$ & $\mathrm{x}$ & $\mathrm{x}$ & $\sqrt{ }$ & $\mathrm{x}$ & $\mathrm{x}$ & $\sqrt{ }$ & $\mathrm{x}$ & $\mathrm{x}$ & $\mathrm{x}$ & $\mathrm{x}$ & $\sqrt{ }$ & $\sqrt{ }$ & $\sqrt{ }$ & $\mathrm{x}$ & $\mathrm{x}$ & $\mathrm{x}$ & $\mathrm{x}$ & $\mathrm{x}$ & $\mathrm{x}$ & $\mathrm{x}$ & $\mathrm{x}$ & $\mathrm{x}$ & $\mathrm{x}$ \\
\hline
\end{tabular}

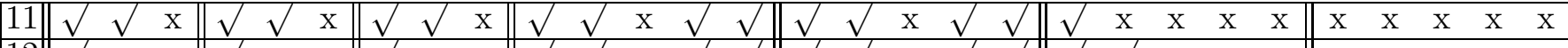

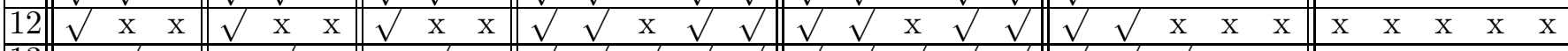

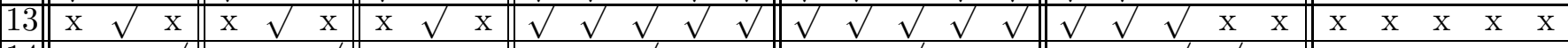
\begin{tabular}{|l||ccc||ccc||ccc||ccccc||cccccc||ccccc||ccccc|}
\hline 14 & $\mathrm{x}$ & $\mathrm{x}$ & $\sqrt{ }$ & $\mathrm{x}$ & $\mathrm{x}$ & $\sqrt{ }$ & $\mathrm{x}$ & $\mathrm{x}$ & $\mathrm{x}$ & $\mathrm{x}$ & $\mathrm{x}$ & $\sqrt{ }$ & $\mathrm{x}$ & $\mathrm{x}$ & $\mathrm{x}$ & $\mathrm{x}$ & $\sqrt{ }$ & $\mathrm{x}$ & $\mathrm{x}$ & $\mathrm{x}$ & $\mathrm{x}$ & $\sqrt{ }$ & $\sqrt{ }$ & $\mathrm{x}$ & $\mathrm{x}$ & $\mathrm{x}$ & $\mathrm{x}$ & $\mathrm{x}$ & $\mathrm{x}$ \\
\hline
\end{tabular} \begin{tabular}{|l||ccc||ccc||ccc||ccccc||cccccc||ccccc||ccccc|}
\hline 15 & $\mathrm{x}$ & $\mathrm{x}$ & $\mathrm{x}$ & $\mathrm{x}$ & $\mathrm{x}$ & $\mathrm{x}$ & $\mathrm{x}$ & $\mathrm{x}$ & $\sqrt{ }$ & $\mathrm{x}$ & $\mathrm{x}$ & $\sqrt{ }$ & $\mathrm{x}$ & $\mathrm{x}$ & $\mathrm{x}$ & $\mathrm{x}$ & $\sqrt{ }$ & $\mathrm{x}$ & $\mathrm{x}$ & $\mathrm{x}$ & $\mathrm{x}$ & $\sqrt{ }$ & $\sqrt{ }$ & $\sqrt{ }$ & $\mathrm{x}$ & $\mathrm{x}$ & $\mathrm{x}$ & $\mathrm{x}$ & $\mathrm{x}$ \\
\hline
\end{tabular}

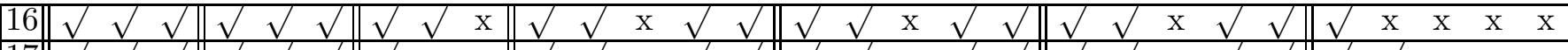
\begin{tabular}{|l|lll|lll||lll||lllll|llllll||llllll||lllll|}
\hline 17 & $\sqrt{ }$ & $\sqrt{ }$ & $\sqrt{ }$ & $\sqrt{ }$ & $\sqrt{ }$ & $\sqrt{ }$ & $\sqrt{ }$ & $\mathrm{x}$ & $\mathrm{x}$ & $\sqrt{ }$ & $\sqrt{ }$ & $\mathrm{x}$ & $\sqrt{ }$ & $\sqrt{ }$ & $\sqrt{ }$ & $\sqrt{ }$ & $\mathrm{x}$ & $\sqrt{ }$ & $\sqrt{ }$ & $\sqrt{ }$ & $\sqrt{ }$ & $\mathrm{x}$ & $\sqrt{ }$ & $\sqrt{ }$ & $\sqrt{ }$ & $\sqrt{ }$ & $\mathrm{x}$ & $\mathrm{x}$ & $\mathrm{x}$ \\
\hline
\end{tabular}

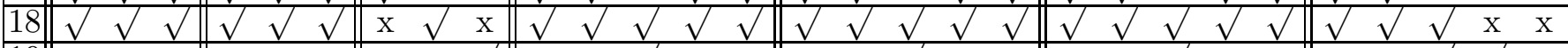

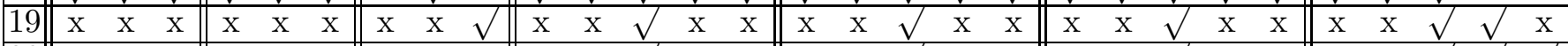
\begin{tabular}{|l||ccc||ccc||ccc||cccccc||ccc|cc||cccccc|ccccc|}
\hline 20 & $\mathrm{x}$ & $\mathrm{x}$ & $\mathrm{x}$ & $\mathrm{x}$ & $\mathrm{x}$ & $\mathrm{x}$ & $\mathrm{x}$ & $\mathrm{x}$ & $\mathrm{x}$ & $\mathrm{x}$ & $\mathrm{x}$ & $\sqrt{ }$ & $\mathrm{x}$ & $\mathrm{x}$ & $\mathrm{x}$ & $\mathrm{x}$ & $\sqrt{ }$ & $\mathrm{x}$ & $\mathrm{x}$ & $\mathrm{x}$ & $\mathrm{x}$ & $\sqrt{ }$ & $\mathrm{x}$ & $\mathrm{x}$ & $\mathrm{x}$ & $\mathrm{x}$ & $\sqrt{ }$ & $\sqrt{ }$ & $\sqrt{ }$ \\
\hline
\end{tabular}

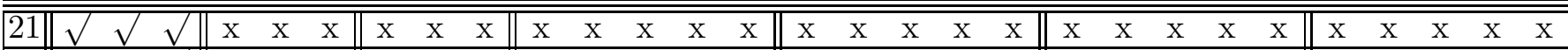
\begin{tabular}{|l|lll||lll||lll||llllll||lllllll||llllll|lllll|}
\hline 22 & $\mathrm{x}$ & $\mathrm{x}$ & $\sqrt{ }$ & $\mathrm{x}$ & $\mathrm{x}$ & $\mathrm{x}$ & $\mathrm{x}$ & $\mathrm{x}$ & $\mathrm{x}$ & $\mathrm{x}$ & $\mathrm{x}$ & $\mathrm{x}$ & $\mathrm{x}$ & $\mathrm{x}$ & $\mathrm{x}$ & $\mathrm{x}$ & $\mathrm{x}$ & $\mathrm{x}$ & $\mathrm{x}$ & $\mathrm{x}$ & $\mathrm{x}$ & $\mathrm{x}$ & $\mathrm{x}$ & $\mathrm{x}$ & $\mathrm{x}$ & $\mathrm{x}$ & $\mathrm{x}$ & $\mathrm{x}$ & $\mathrm{x}$ \\
\hline
\end{tabular} \begin{tabular}{||l|lll||lll||ccc||ccccc||ccccc||ccccc||ccccc|}
\hline 23 & $\mathrm{x}$ & $\sqrt{ }$ & $\sqrt{ }$ & $\mathrm{x}$ & $\mathrm{x}$ & $\mathrm{x}$ & $\mathrm{x}$ & $\mathrm{x}$ & $\mathrm{x}$ & $\mathrm{x}$ & $\mathrm{x}$ & $\mathrm{x}$ & $\mathrm{x}$ & $\mathrm{x}$ & $\mathrm{x}$ & $\mathrm{x}$ & $\mathrm{x}$ & $\mathrm{x}$ & $\mathrm{x}$ & $\mathrm{x}$ & $\mathrm{x}$ & $\mathrm{x}$ & $\mathrm{x}$ & $\mathrm{x}$ & $\mathrm{x}$ & $\mathrm{x}$ & $\mathrm{x}$ & $\mathrm{x}$ & $\mathrm{x}$ \\
\hline
\end{tabular} \begin{tabular}{|l|lll||lll||lll||llllll||llllll||llllll||lllll|}
\hline 24 & $\sqrt{ }$ & $\mathrm{x}$ & $\sqrt{ }$ & $\mathrm{x}$ & $\mathrm{x}$ & $\mathrm{x}$ & $\mathrm{x}$ & $\mathrm{x}$ & $\mathrm{x}$ & $\mathrm{x}$ & $\mathrm{x}$ & $\mathrm{x}$ & $\mathrm{x}$ & $\mathrm{x}$ & $\mathrm{x}$ & $\mathrm{x}$ & $\mathrm{x}$ & $\mathrm{x}$ & $\mathrm{x}$ & $\mathrm{x}$ & $\mathrm{x}$ & $\mathrm{x}$ & $\mathrm{x}$ & $\mathrm{x}$ & $\mathrm{x}$ & $\mathrm{x}$ & $\mathrm{x}$ & $\mathrm{x}$ & $\mathrm{x}$ \\
\hline
\end{tabular} \begin{tabular}{|l|lll||lll||lll||llllll||llllll||llllll||lllll|}
\hline 25 & $\mathrm{x}$ & $\sqrt{ }$ & $\sqrt{ }$ & $\mathrm{x}$ & $\mathrm{x}$ & $\mathrm{x}$ & $\mathrm{x}$ & $\mathrm{x}$ & $\mathrm{x}$ & $\mathrm{x}$ & $\mathrm{x}$ & $\mathrm{x}$ & $\mathrm{x}$ & $\mathrm{x}$ & $\mathrm{x}$ & $\mathrm{x}$ & $\mathrm{x}$ & $\mathrm{x}$ & $\mathrm{x}$ & $\mathrm{x}$ & $\mathrm{x}$ & $\mathrm{x}$ & $\mathrm{x}$ & $\mathrm{x}$ & $\mathrm{x}$ & $\mathrm{x}$ & $\mathrm{x}$ & $\mathrm{x}$ & $\mathrm{x}$ \\
\hline
\end{tabular} \begin{tabular}{|l||ll||lll||lll||llllll||llllll||lllll|lllll|}
\hline 26 & $\sqrt{ }$ & $\mathrm{x}$ & $\sqrt{ }$ & $\mathrm{x}$ & $\mathrm{x}$ & $\mathrm{x}$ & $\mathrm{x}$ & $\mathrm{x}$ & $\mathrm{x}$ & $\mathrm{x}$ & $\mathrm{x}$ & $\mathrm{x}$ & $\mathrm{x}$ & $\mathrm{x}$ & $\mathrm{x}$ & $\mathrm{x}$ & $\mathrm{x}$ & $\mathrm{x}$ & $\mathrm{x}$ & $\mathrm{x}$ & $\mathrm{x}$ & $\mathrm{x}$ & $\mathrm{x}$ & $\mathrm{x}$ & $\mathrm{x}$ & $\mathrm{x}$ & $\mathrm{x}$ & $\mathrm{x}$ & $\mathrm{x}$ \\
\hline
\end{tabular}

\begin{tabular}{|l||ccc||ccc||ccc||ccccc||cccccc||ccccc||ccccc|}
\hline 27 & $\mathrm{x}$ & $\mathrm{x}$ & $\mathrm{x}$ & $\sqrt{ }$ & $\sqrt{ }$ & $\sqrt{ }$ & $\mathrm{x}$ & $\mathrm{x}$ & $\mathrm{x}$ & $\mathrm{x}$ & $\mathrm{x}$ & $\mathrm{x}$ & $\mathrm{x}$ & $\mathrm{x}$ & $\mathrm{x}$ & $\mathrm{x}$ & $\mathrm{x}$ & $\mathrm{x}$ & $\mathrm{x}$ & $\mathrm{x}$ & $\mathrm{x}$ & $\mathrm{x}$ & $\mathrm{x}$ & $\mathrm{x}$ & $\mathrm{x}$ & $\mathrm{x}$ & $\mathrm{x}$ & $\mathrm{x}$ & $\mathrm{x}$ \\
\hline
\end{tabular}

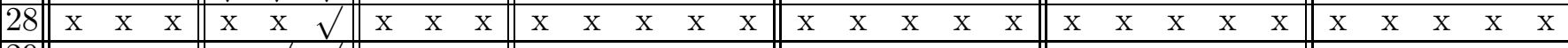

\begin{tabular}{|l|lll||lll||lll||lllll||lllll||llllll||lllll|}
\hline 29 & $\mathrm{x}$ & $\mathrm{x}$ & $\mathrm{x}$ & $\mathrm{x}$ & $\sqrt{ }$ & $\sqrt{ }$ & $\mathrm{x}$ & $\mathrm{x}$ & $\mathrm{x}$ & $\mathrm{x}$ & $\mathrm{x}$ & $\mathrm{x}$ & $\mathrm{x}$ & $\mathrm{x}$ & $\mathrm{x}$ & $\mathrm{x}$ & $\mathrm{x}$ & $\mathrm{x}$ & $\mathrm{x}$ & $\mathrm{x}$ & $\mathrm{x}$ & $\mathrm{x}$ & $\mathrm{x}$ & $\mathrm{x}$ & $\mathrm{x}$ & $\mathrm{x}$ & $\mathrm{x}$ & $\mathrm{x}$ & $\mathrm{x}$ \\
\hline
\end{tabular}

\begin{tabular}{|l|lll||lll||lll||llllll||llllll||lllll||lllll|}
\hline 30 & $\mathrm{x}$ & $\mathrm{x}$ & $\mathrm{x}$ & $\sqrt{ }$ & $\mathrm{x}$ & $\sqrt{ }$ & $\mathrm{x}$ & $\mathrm{x}$ & $\mathrm{x}$ & $\mathrm{x}$ & $\mathrm{x}$ & $\mathrm{x}$ & $\mathrm{x}$ & $\mathrm{x}$ & $\mathrm{x}$ & $\mathrm{x}$ & $\mathrm{x}$ & $\mathrm{x}$ & $\mathrm{x}$ & $\mathrm{x}$ & $\mathrm{x}$ & $\mathrm{x}$ & $\mathrm{x}$ & $\mathrm{x}$ & $\mathrm{x}$ & $\mathrm{x}$ & $\mathrm{x}$ & $\mathrm{x}$ & $\mathrm{x}$ \\
\hline & $\mathrm{x}$ & $\mathrm{x}$ & $\mathrm{x}$ & $\mathrm{x}$ & & & & $\mathrm{x}$ & $\mathrm{x}$ & $\mathrm{x}$ & $\mathrm{x}$ & $\mathrm{x}$ & $\mathrm{x}$ & $\mathrm{x}$ & $\mathrm{x}$ & $\mathrm{x}$ & $\mathrm{x}$ & $\mathrm{x}$ & $\mathrm{x}$ & $\mathrm{x}$ & $\mathrm{x}$ & $\mathrm{x}$ & $\mathrm{x}$ & $\mathrm{x}$ & $\mathrm{x}$ & $\mathrm{x}$ & $\mathrm{x}$ & $\mathrm{x}$ & $\mathrm{x}$ & $\mathrm{x}$ \\
\hline
\end{tabular}

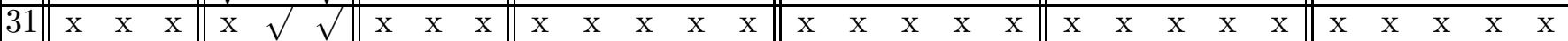

\begin{tabular}{|l|lll||lll||lll||llllll||llllll||lllll||lllll|}
\hline 32 & $\mathrm{x}$ & $\mathrm{x}$ & $\mathrm{x}$ & $\sqrt{ }$ & $\mathrm{x}$ & $\sqrt{ }$ & $\mathrm{x}$ & $\mathrm{x}$ & $\mathrm{x}$ & $\mathrm{x}$ & $\mathrm{x}$ & $\mathrm{x}$ & $\mathrm{x}$ & $\mathrm{x}$ & $\mathrm{x}$ & $\mathrm{x}$ & $\mathrm{x}$ & $\mathrm{x}$ & $\mathrm{x}$ & $\mathrm{x}$ & $\mathrm{x}$ & $\mathrm{x}$ & $\mathrm{x}$ & $\mathrm{x}$ & $\mathrm{x}$ & $\mathrm{x}$ & $\mathrm{x}$ & $\mathrm{x}$ & $\mathrm{x}$ \\
\hline
\end{tabular}

\begin{tabular}{||c||cc||ccc||ccc||ccccc||cccccc||ccccc||ccccc|}
\hline 33 & $\mathrm{x}$ & $\mathrm{x}$ & $\mathrm{x}$ & $\mathrm{x}$ & $\mathrm{x}$ & $\mathrm{x}$ & $\sqrt{ }$ & $\sqrt{ }$ & $\sqrt{ }$ & $\mathrm{x}$ & $\mathrm{x}$ & $\mathrm{x}$ & $\mathrm{x}$ & $\mathrm{x}$ & $\mathrm{x}$ & $\mathrm{x}$ & $\mathrm{x}$ & $\mathrm{x}$ & $\mathrm{x}$ & $\mathrm{x}$ & $\mathrm{x}$ & $\mathrm{x}$ & $\mathrm{x}$ & $\mathrm{x}$ & $\mathrm{x}$ & $\mathrm{x}$ & $\mathrm{x}$ & $\mathrm{x}$ & $\mathrm{x}$ \\
\hline
\end{tabular}

\begin{tabular}{|l|lll||ccc||ccc||ccccc||ccccc||ccccc||ccccc|}
\hline 34 & $\mathrm{x}$ & $\mathrm{x}$ & $\mathrm{x}$ & $\mathrm{x}$ & $\mathrm{x}$ & $\mathrm{x}$ & $\mathrm{x}$ & $\mathrm{x}$ & $\sqrt{ }$ & $\mathrm{x}$ & $\mathrm{x}$ & $\mathrm{x}$ & $\mathrm{x}$ & $\mathrm{x}$ & $\mathrm{x}$ & $\mathrm{x}$ & $\mathrm{x}$ & $\mathrm{x}$ & $\mathrm{x}$ & $\mathrm{x}$ & $\mathrm{x}$ & $\mathrm{x}$ & $\mathrm{x}$ & $\mathrm{x}$ & $\mathrm{x}$ & $\mathrm{x}$ & $\mathrm{x}$ & $\mathrm{x}$ & $\mathrm{x}$ \\
\hline
\end{tabular}

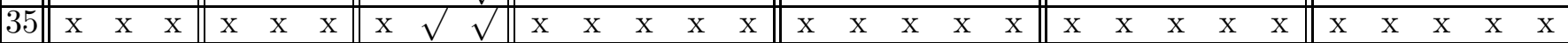

\begin{tabular}{||c|ccc||ccc||ccc||ccccc||ccccc||ccccc|ccccc|}
\hline 36 & $\mathrm{x}$ & $\mathrm{x}$ & $\mathrm{x}$ & $\mathrm{x}$ & $\mathrm{x}$ & $\mathrm{x}$ & $\sqrt{ }$ & $\mathrm{x}$ & $\sqrt{ }$ & $\mathrm{x}$ & $\mathrm{x}$ & $\mathrm{x}$ & $\mathrm{x}$ & $\mathrm{x}$ & $\mathrm{x}$ & $\mathrm{x}$ & $\mathrm{x}$ & $\mathrm{x}$ & $\mathrm{x}$ & $\mathrm{x}$ & $\mathrm{x}$ & $\mathrm{x}$ & $\mathrm{x}$ & $\mathrm{x}$ & $\mathrm{x}$ & $\mathrm{x}$ & $\mathrm{x}$ & $\mathrm{x}$ & $\mathrm{x}$ \\
\hline
\end{tabular} \begin{tabular}{|l|lll||ccc||ccc||ccccc||cccccc||ccccc||ccccc|}
\hline 37 & $\mathrm{x}$ & $\mathrm{x}$ & $\mathrm{x}$ & $\mathrm{x}$ & $\mathrm{x}$ & $\mathrm{x}$ & $\mathrm{x}$ & $\sqrt{ }$ & $\sqrt{ }$ & $\mathrm{x}$ & $\mathrm{x}$ & $\mathrm{x}$ & $\mathrm{x}$ & $\mathrm{x}$ & $\mathrm{x}$ & $\mathrm{x}$ & $\mathrm{x}$ & $\mathrm{x}$ & $\mathrm{x}$ & $\mathrm{x}$ & $\mathrm{x}$ & $\mathrm{x}$ & $\mathrm{x}$ & $\mathrm{x}$ & $\mathrm{x}$ & $\mathrm{x}$ & $\mathrm{x}$ & $\mathrm{x}$ & $\mathrm{x}$ \\
\hline
\end{tabular} \begin{tabular}{|l||ll||lll||lll||llllll||llllll||lllll||lllll|}
\hline 38 & $\mathrm{x}$ & $\mathrm{x}$ & $\mathrm{x}$ & $\mathrm{x}$ & $\mathrm{x}$ & $\mathrm{x}$ & $\sqrt{ }$ & $\mathrm{x}$ & $\sqrt{ }$ & $\mathrm{x}$ & $\mathrm{x}$ & $\mathrm{x}$ & $\mathrm{x}$ & $\mathrm{x}$ & $\mathrm{x}$ & $\mathrm{x}$ & $\mathrm{x}$ & $\mathrm{x}$ & $\mathrm{x}$ & $\mathrm{x}$ & $\mathrm{x}$ & $\mathrm{x}$ & $\mathrm{x}$ & $\mathrm{x}$ & $\mathrm{x}$ & $\mathrm{x}$ & $\mathrm{x}$ & $\mathrm{x}$ & $\mathrm{x}$ \\
\hline
\end{tabular}

\begin{tabular}{|l||ccc||ccc||ccc||cccc||cccccc||ccccc||ccccc|}
\hline \hline 39 & $\mathrm{x}$ & $\mathrm{x}$ & $\mathrm{x}$ & $\mathrm{x}$ & $\mathrm{x}$ & $\mathrm{x}$ & $\mathrm{x}$ & $\mathrm{x}$ & $\mathrm{x}$ & $\mathrm{x}$ & $\mathrm{x}$ & $\mathrm{x}$ & $\sqrt{ }$ & $\sqrt{ }$ & $\mathrm{x}$ & $\mathrm{x}$ & $\mathrm{x}$ & $\mathrm{x}$ & $\mathrm{x}$ & $\mathrm{x}$ & $\mathrm{x}$ & $\mathrm{x}$ & $\mathrm{x}$ & $\mathrm{x}$ & $\mathrm{x}$ & $\mathrm{x}$ & $\mathrm{x}$ & $\mathrm{x}$ & $\mathrm{x}$ \\
\hline
\end{tabular} \begin{tabular}{|l|lll||lll||lll||llllll||llllll||llllll||lllll|}
\hline 40 & $\mathrm{x}$ & $\mathrm{x}$ & $\mathrm{x}$ & $\mathrm{x}$ & $\mathrm{x}$ & $\mathrm{x}$ & $\mathrm{x}$ & $\mathrm{x}$ & $\mathrm{x}$ & $\mathrm{x}$ & $\sqrt{ }$ & $\mathrm{x}$ & $\mathrm{x}$ & $\sqrt{ }$ & $\mathrm{x}$ & $\mathrm{x}$ & $\mathrm{x}$ & $\mathrm{x}$ & $\mathrm{x}$ & $\mathrm{x}$ & $\mathrm{x}$ & $\mathrm{x}$ & $\mathrm{x}$ & $\mathrm{x}$ & $\mathrm{x}$ & $\mathrm{x}$ & $\mathrm{x}$ & $\mathrm{x}$ & $\mathrm{x}$ \\
\hline
\end{tabular}

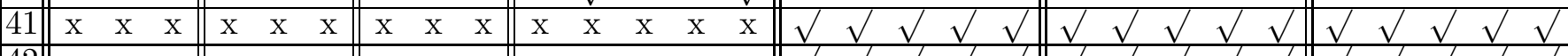

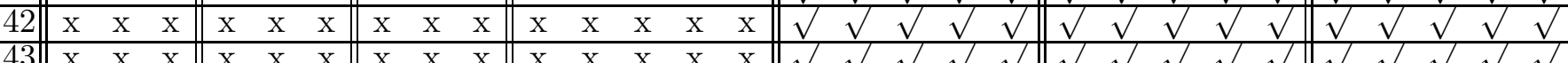

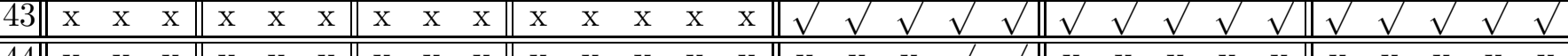

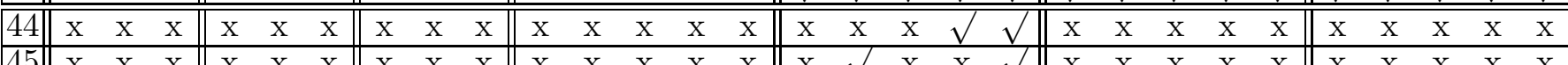
\begin{tabular}{|l|ccc||ccc||ccc||cccccc||ccccccc||ccccc||cccccc|}
\hline 45 & $\mathrm{x}$ & $\mathrm{x}$ & $\mathrm{x}$ & $\mathrm{x}$ & $\mathrm{x}$ & $\mathrm{x}$ & $\mathrm{x}$ & $\mathrm{x}$ & $\mathrm{x}$ & $\mathrm{x}$ & $\mathrm{x}$ & $\mathrm{x}$ & $\mathrm{x}$ & $\mathrm{x}$ & $\mathrm{x}$ & $\sqrt{ }$ & $\mathrm{x}$ & $\mathrm{x}$ & $\sqrt{ }$ & $\mathrm{x}$ & $\mathrm{x}$ & $\mathrm{x}$ & $\mathrm{x}$ & $\mathrm{x}$ & $\mathrm{x}$ & $\mathrm{x}$ & $\mathrm{x}$ & $\mathrm{x}$ & $\mathrm{x}$ \\
\hline 46 & $\mathrm{x}$ & $\mathrm{x}$ & $\mathrm{x}$ & $\mathrm{x}$ & $\mathrm{x}$ & $\mathrm{x}$ & $\mathrm{x}$ & $\mathrm{x}$ & $\mathrm{x}$ & $\mathrm{x}$ & $\mathrm{x}$ & $\mathrm{x}$ & $\mathrm{x}$ & $\mathrm{x}$ & $\mathrm{x}$ & $\mathrm{x}$ & $\mathrm{x}$ & $\mathrm{x}$ & $\mathrm{x}$ & $\sqrt{ }$ & $\sqrt{ }$ & $\sqrt{ }$ & $\sqrt{ }$ & $\sqrt{ }$ & $\sqrt{ }$ & $\sqrt{ }$ & $\sqrt{ }$ & $\sqrt{ }$ & $\sqrt{ }$ \\
\hline
\end{tabular}

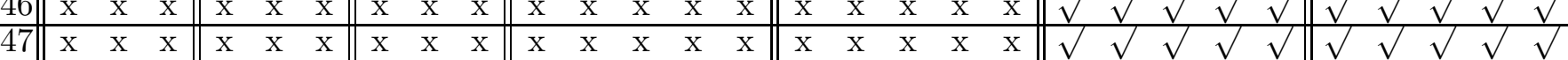

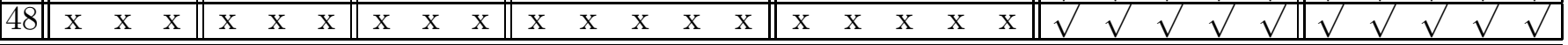

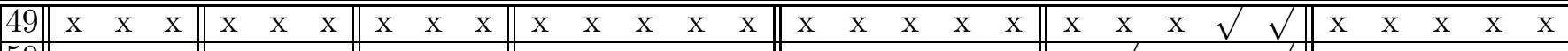

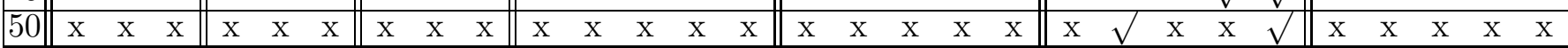

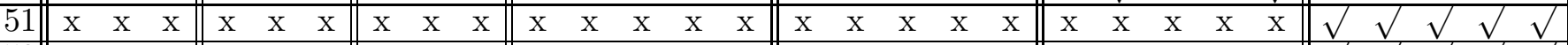
\begin{tabular}{|l||ccc||ccc||ccc||ccccc||ccccc||ccccc||lllll|}
\hline 52 & $\mathrm{x}$ & $\mathrm{x}$ & $\mathrm{x}$ & $\mathrm{x}$ & $\mathrm{x}$ & $\mathrm{x}$ & $\mathrm{x}$ & $\mathrm{x}$ & $\mathrm{x}$ & $\mathrm{x}$ & $\mathrm{x}$ & $\mathrm{x}$ & $\mathrm{x}$ & $\mathrm{x}$ & $\mathrm{x}$ & $\mathrm{x}$ & $\mathrm{x}$ & $\mathrm{x}$ & $\mathrm{x}$ & $\mathrm{x}$ & $\mathrm{x}$ & $\mathrm{x}$ & $\mathrm{x}$ & $\mathrm{x}$ & $\sqrt{ }$ & $\sqrt{ }$ & $\sqrt{ }$ & $\sqrt{ }$ & $\sqrt{ }$ \\
\hline 53 & $\mathrm{x}$ & $\mathrm{x}$ & $\mathrm{x}$ & $\mathrm{x}$ & $\mathrm{x}$ & $\mathrm{x}$ & $\mathrm{x}$ & $\mathrm{x}$ & $\mathrm{x}$ & $\mathrm{x}$ & $\mathrm{x}$ & $\mathrm{x}$ & $\mathrm{x}$ & $\mathrm{x}$ & $\mathrm{x}$ & $\mathrm{x}$ & $\mathrm{x}$ & $\mathrm{x}$ & $\mathrm{x}$ & $\mathrm{x}$ & $\mathrm{x}$ & $\mathrm{x}$ & $\mathrm{x}$ & $\mathrm{x}$ & $\sqrt{ }$ & & & & \\
\hline
\end{tabular} \begin{tabular}{|l|lll||lll||ccc||ccccc||ccccc||ccccc||lllll|}
53 & $\mathrm{x}$ & $\mathrm{x}$ & $\mathrm{x}$ & $\mathrm{x}$ & $\mathrm{x}$ & $\mathrm{x}$ & $\mathrm{x}$ & $\mathrm{x}$ & $\mathrm{x}$ & $\mathrm{x}$ & $\mathrm{x}$ & $\mathrm{x}$ & $\mathrm{x}$ & $\mathrm{x}$ & $\mathrm{x}$ & $\mathrm{x}$ & $\mathrm{x}$ & $\mathrm{x}$ & $\mathrm{x}$ & $\mathrm{x}$ & $\mathrm{x}$ & $\mathrm{x}$ & $\mathrm{x}$ & $\mathrm{x}$ & $\sqrt{ }$ & $\sqrt{ }$ & $\sqrt{ }$ & $\sqrt{ }$ & $\sqrt{ }$ \\
\hline
\end{tabular} \begin{tabular}{|l||lll||lll||ccc||ccccc||ccccc||ccccc||ccccc|}
\hline 54 & $\mathrm{x}$ & $\mathrm{x}$ & $\mathrm{x}$ & $\mathrm{x}$ & $\mathrm{x}$ & $\mathrm{x}$ & $\mathrm{x}$ & $\mathrm{x}$ & $\mathrm{x}$ & $\mathrm{x}$ & $\mathrm{x}$ & $\mathrm{x}$ & $\mathrm{x}$ & $\mathrm{x}$ & $\mathrm{x}$ & $\mathrm{x}$ & $\mathrm{x}$ & $\mathrm{x}$ & $\mathrm{x}$ & $\mathrm{x}$ & $\mathrm{x}$ & $\mathrm{x}$ & $\mathrm{x}$ & $\mathrm{x}$ & $\mathrm{x}$ & $\mathrm{x}$ & $\mathrm{x}$ & $\sqrt{ }$ & $\sqrt{ }$ \\
\hline
\end{tabular} \begin{tabular}{|l|lll||lll||lll||ccccc||ccccc||ccccc||ccccc|}
\hline 55 & $\mathrm{x}$ & $\mathrm{x}$ & $\mathrm{x}$ & $\mathrm{x}$ & $\mathrm{x}$ & $\mathrm{x}$ & $\mathrm{x}$ & $\mathrm{x}$ & $\mathrm{x}$ & $\mathrm{x}$ & $\mathrm{x}$ & $\mathrm{x}$ & $\mathrm{x}$ & $\mathrm{x}$ & $\mathrm{x}$ & $\mathrm{x}$ & $\mathrm{x}$ & $\mathrm{x}$ & $\mathrm{x}$ & $\mathrm{x}$ & $\mathrm{x}$ & $\mathrm{x}$ & $\mathrm{x}$ & $\mathrm{x}$ & $\mathrm{x}$ & $\sqrt{ }$ & $\mathrm{x}$ & $\mathrm{x}$ & $\sqrt{ }$ \\
\hline \hline
\end{tabular}

Table 8: $P(\phi)$ for $\phi=\left(x_{1} \vee x_{2} \vee x_{3}\right) \wedge\left(\bar{x}_{1} \vee \bar{x}_{2} \vee x_{3}\right) \wedge\left(\bar{x}_{2} \vee \bar{x}_{3} \vee x_{4}\right)$ and the coding example in Section 4. 